\title{
Aerodynamic Challenges for the Mars Science Laboratory Entry Descent and Landing
}

\author{
Mark Schoenenberger*, \\ Artem Dyakonov ${ }^{\dagger}$ \\ Pieter Buning; \\ NASA Langley Research Center \\ William Scallion\$ \\ and John Van Norman, \\ Analytical Mechanics Associates Inc.
}

\begin{abstract}
An overview of several important aerodynamics challenges new to the Mars Science Laboratory (MSL) entry vehicle are presented. The MSL entry capsule is a $70^{\circ}$ sphere cone-based on the original Mars Viking entry capsule. Due to payload and landing accuracy requirements, MSL will be flying at the highest lift-to-drag ratio of any capsule sent to Mars $(\mathrm{L} / \mathrm{D}=\mathbf{0 . 2 4})$. The capsule will also be flying a guided entry, performing bank maneuvers, a first for Mars entry. The system's mechanical design and increased performance requirements require an expansion of the MSL flight envelope beyond those of historical missions. In certain areas, the experience gained by Viking and other recent Mars missions can no longer be claimed as heritage information. New analysis and testing is required to ensure the safe flight of the MSL entry vehicle. The challenge topics include: hypersonic gas chemistry and laminar-versus-turbulent flow effects on trim angle, a general risk assessment of flying at greater angles-of-attack than Viking, quantifying the aerodynamic interactions induced by a new reaction control system and a risk assessment of recontact of a series of masses jettisoned prior to parachute deploy. An overview of the analysis and tests being conducted to understand and reduce risk in each of these areas is presented.The need for proper modeling and implementation of uncertainties for use in trajectory simulation has resulted in a revision of prior models and additional analysis for the MSL entry vehicle. The six degree-of-freedom uncertainty model and new analysis to quantify roll torque dispersions are presented.
\end{abstract}

\footnotetext{
*Atmospheric Flight and Entry Systems Branch, member AIAA

${ }^{\dagger}$ Atmospheric Flight and Entry Systems Branch, member AIAA

${ }^{\ddagger}$ Computational Aerosciences Branch, member AIAA

$\S$ Aerothermodynamics Branch, member AIAA

『Atmospheric Flight and Entry Systems Branch, member AIAA
} 


\section{Nomenclature}

\begin{tabular}{llll}
$A$ & Nozzle Area & $v$ & Balance mass "volume" \\
$C_{A}$ & Axial force coefficient & $x, y, z$ & Entry vehicle body coordinates \\
$C_{B}$ & Ballistic coefficient & $y$ & Height above surface \\
$C_{D}$ & Drag coefficient & & \\
$C_{l}$ & Rolling moment coefficient & Greek & \\
$C_{m}$ & Pitching moment coefficient & $\alpha$ & Angle of attack \\
$C_{N}$ & Normal force coefficient & $\beta$ & Angle of sideslip \\
$C_{n}$ & Yawing moment coefficient & $\gamma$ & Ratio of specific heats \\
$C_{p}$ & Pressure coefficient & $\delta^{*}$ & Displacement thickness \\
$C_{Y}$ & Side force coefficient & $\rho$ & Density \\
$D$ & Drag & Subscripts & \\
$d$ & Reference diameter & $b$ & Backshell \\
$h$ & Reference height & $c$ & RCS nozzle chamber \\
$L$ & Lift & $c g$ & Center of gravity \\
$l$ & Length & Disp & Dispersed value \\
$M$ & Mach number & $e$ & Nozzle exit conditions \\
$m$ & Mass & $n$ & Normal to step \\
$\dot{m}$ & Mass flow rate & $T$ & Total \\
$P$ & Pressure & Trim & Trimmed attitude \\
$\bar{q}$ & Mean dynamic pressure & $\infty$ & Freestream conditions \\
$S$ & Reference area & Superscripts & \\
$U$ & Uncertainty variable & $A$ & Adder \\
$V$ & Velocity & $M$ & Multiplier \\
& & & \\
\hline & & &
\end{tabular}

\section{Introduction}

The Mars Science Laboratory mission will deliver a $900 \mathrm{~kg}$ rover to the surface of Mars in 2012. This will be the largest payload ever landed on the red planet. Consequently, the MSL entry capsule will be the largest flown to Mars (4.5 $\mathrm{m}$ diameter) and most massive ( $\sim 2800 \mathrm{~kg}$ entry mass), with the largest ballistic coefficient $\left(B_{E V}=110\right)$. The entry capsule must fly a guided, lifting trajectory to a land the rover within a prescribed landing ellipse with a major axis of $10 \mathrm{~km}$. The entry vehicle (EV) lift is also required to arrive at supersonic parachute deploy conditions with sufficient altitude to execute deployment, deceleration and the remaining separation events and powered descent phase to touchdown. During powered descent, the lander will drop the rover on a tether in the "skycrane" mode, touching down on Mars with negligible vertical and horizontal velocities.

To achieve the highest performance guidance capabilities with such a massive EV, the MSL aerodynamic envelope must be expanded beyond those of earlier vehicles. This paper provides an overview of the additional aerodynamic challenges identified as MSL expands the flight envelope, a summary of how the challenges are being addressed through testing and analysis, and the current status of ongoing work. Test and analysis reports with more detailed descriptions of findings summarized here will be (or have been) published as individual papers at a later date.

\section{A. Viking and configuration heritage}

The MSL entry capsule outer mold line (OML) is based on the Mars Viking entry vehicle, designed and developed in the 1970's. All US entry capsules since Viking have used the same $70^{\circ}$ forebody shape, while the backshell shapes have varied slightly to accommodate payload packaging considerations. Figure 1 shows the Viking and MSL configurations. Some notable differences include the increased shoulder radius on the MSL forebody, the elongated biconic backshell section, and the addition of a third truncated cone on the 
backshell which holds MSL's parachute canister. Also note that the Viking diameter was $3.5 m$, considerably smaller than MSL.
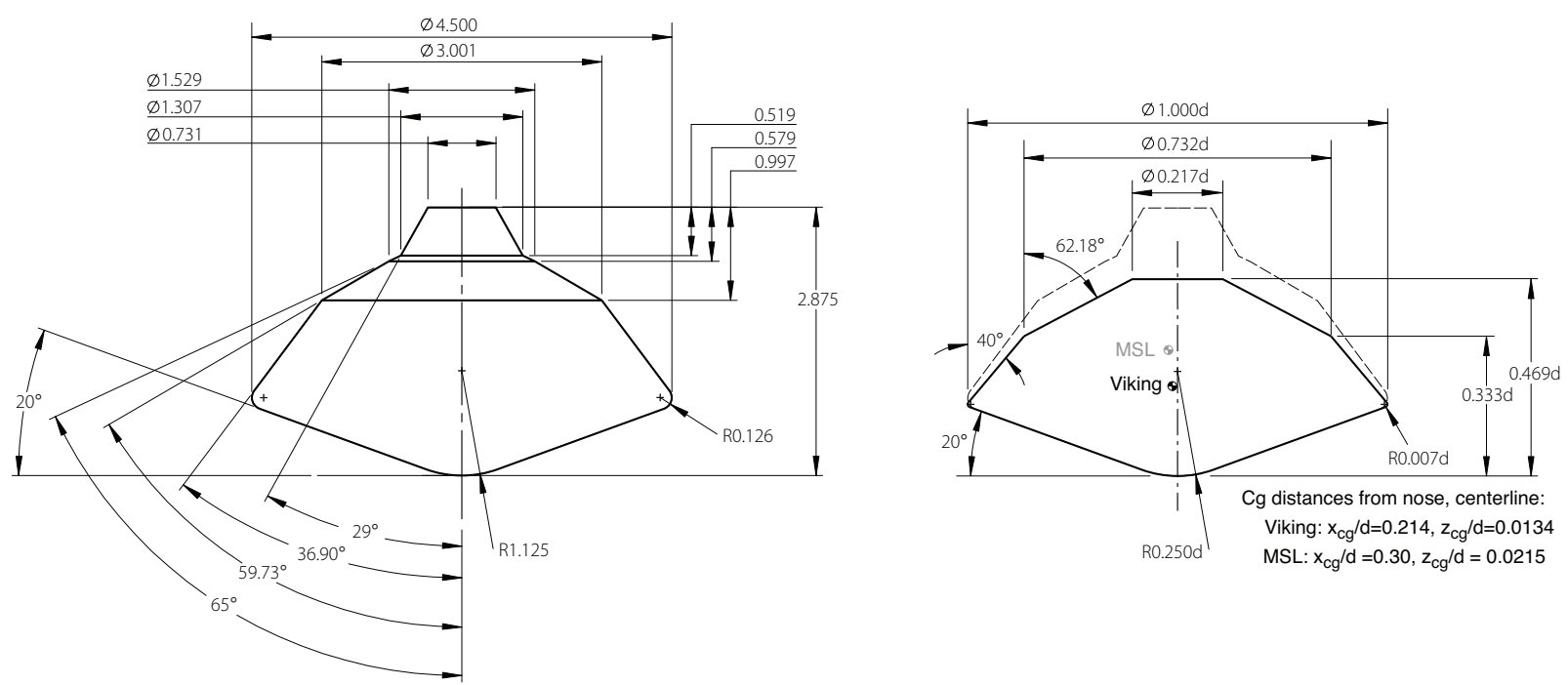

Figure 1. MSL and Viking geometries, Viking/MSL comparison scaled to unit diameter

The basic forebody geometry has been kept the same as the original Viking shape to benefit from the somewhat nebulous concept of "heritage." The successful touchdown of the Viking landers demonstrated that a $70^{\circ}$ sphere-cone EV is capable of entry and descent through the Martian atmosphere. Ground based tests and analyses at the time adequately predicted the aerodynamic behavior for entry profile design and landing ellipse predictions. The Viking missions validate a large number of tests and methodologies from which MSL can now benefit, either using old Viking data directly or basing design decisions on lessons learned by Viking. Using the capsule geometry of past missions reduces risk and the expense of developing a new aerodynamic shape. However, as the MSL configuration or flight profile deviates from Viking, the claim of benefit due to heritage must be challenged and proven. The MSL vehicle is heavier, larger, has OML changes, a different RCS configuration, will trim at a larger angle-of-attack than Viking, will maneuver, and will jettison mass during flight. All of these changes must be evaluated with analysis and/or testing to ensure the changes do not cause the MSL EV to fly different than Viking. For each design change, some Viking heritage is lost and new data must be collected to retire the risks introduced by the addition of new failure modes.

The design philosophy of MSL (much like recent Mars missions) can be summarized as follows: Viking is the validated point of departure with a body of knowledge and experience that gives MSL a good head start. New computational fluid dynamics (CFD) analysis capabilities have increased the confidence in hypersonic aerodynamic predictions where real gas effects are significant. These analyses have been validated by comparisons with Viking wind tunnel data as well as flight performance of Mars Pathfinder (MPF), Mars Exploration Rovers (MER) and Mars Phoenix (MPX). The analyses performed, experimental data collected and understanding gained from the reconstruction of previous missions make up the heritage information upon which a new mission bases its risk assessment. As new mission constraints result in configuration changes, the impact on flight performance must be investigated and quantified rigorously to ensure that the vehicle can still be flown safely. As MSL will be lifting during entry, the project must weigh the Viking experience more heavily than it might have if it were flying ballistically; where MPF, MER and MPX would provide more relevant validation data and more modern supporting analyses. In some instances, computational methods used for the recent Mars missions are not validated for use on a lifting vehicle and additional analysis is required. This paper gives an overview of the significant changes from the Viking entry vehicle, how the changes were analyzed, and general conclusions drawn from the analyses. 


\section{B. MSL entry}

Figure 2 shows a representative MSL entry trajectory compared to all previous successful Mars landings. MSL will dive deeper into the atmosphere than previous missions, using the increased lift force at lower altitudes (greater density) to fly a guided entry to a precise landing ellipse. During each phase of entry down to parachute deploy, MSL is is expanding the flight envelope for a $70^{\circ}$ sphere cone. The following briefly summarizes the events during entry and the new challenges identified in each flight regime.

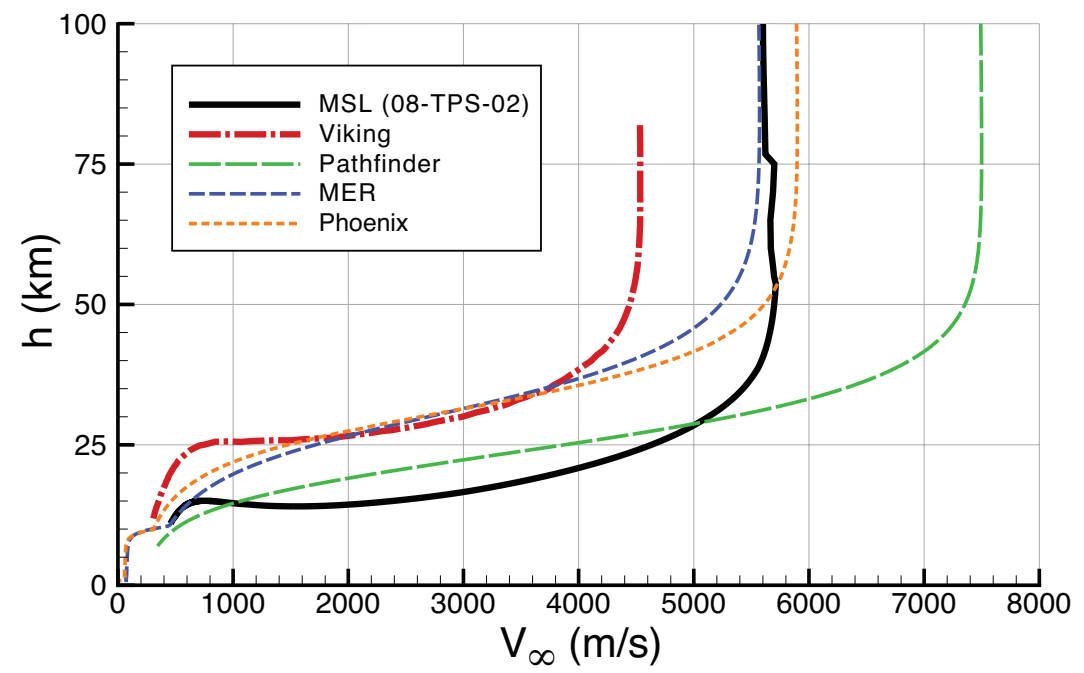

Figure 2. MSL entry trajectory, compared with previous Mars missions

\section{Hypersonic Regime}

The MSL entry vehicle will enter the Martian atmosphere traveling at $5500 \mathrm{~m} / \mathrm{s}(\sim$ Mach 30$)$. The aeroshell does most of the deceleration work, slowing the EV down to low supersonic speeds just prior to parachute deploy.

In the hypersonic regime, chemical reaction rates within the shock layer ahead of the vehicle changes from frozen flow, to non-equilibrium to equilibrium along the trajectory. The chemical reactions of $\mathrm{CO}_{2}$ and other gases and how those reactions change with velocity and density cause the static aerodynamic coefficients to vary somewhat in the hypersonic regime. To accurately predict the MSL trim angle-of-attack and drag, chemically reacting CFD predictions are used to model all hypersonic aerodynamics. The accurate prediction of trim angle is necessary to predict the lifting capability of the EV as well as provide the proper conditions for analysis of the thermal protection system at peak heating conditions.

The EV will enter at a total angle of attack of approximately $-16^{\circ}$. The vehicle is flying at a lifting angle-of-attack to perform guidance maneuvers by controlling orientation of the lift vector. Blunt bodies require a negative angle-of-attack for positive lift, as the pointing of the axial force relative to the freestream flow produces the majority of the lift force acting on the vehicle. The normal force is negative at a negative angle of attack, but is small compared to the axial force component of lift. The capsule uses an RCS system to perform a series of bank reversals, rotating the lift vector to manage energy. This energy management guides the vehicle to the desired landing site, concerned first with reaching the desired down-range location while also trying to maximize the altitude at which the parachute deploys.

Viking base pressure measurements supported by CFD analysis indicates that backshell pressure peaks just after peak dynamic pressure in the hypersonic regime. This high pressure provides the most adverse environment for any aero/RCS interactions. The RCS configuration differs from Viking, so the question of aero/RCS interaction demands special attention. 
The controller that commands the RCS system to perform bank maneuvers (and damp unwanted oscillations) is tuned using nominal aerodynamic coefficients linearized about the predicted trim angle-of-attack. In addition to aero/RCS interactions, if the actual flight deviates from that predicted by the aerodynamic database, there is a possibility that the controller might fail. Trajectory simulations have shown that the controller can have problems following the guidance path if there is a persistent roll torque acting on the capsule while performing bank reversals at or near peak dynamic pressure. The quantification of the aerodynamic uncertainties is critical to understanding the performance of the controller, with roll torque uncertainties being of particular interest.

\section{Supersonic Regime}

After managing energy through the region of peak dynamic pressure, the guidance algorithm begins to steer the vehicle to the proper heading to reach the center of the desired landing ellipse. This is again achieved with the RCS system banking the vehicle and using the lift vector to change heading. A high parachute deploy altitude is also desired for sufficient timeline to complete powered descent. The controller balances the desire for altitude and correct heading in its commanded maneuvers. As the EV slows towards Mach one, the base pressure again begins to increase (after a local minimum near Mach 6). Non-lifting missions have shown that dynamic instabilities driven by wake flow appear near Mach 3.5, and become increasingly unstable down to parachute deploy conditions. The combination of maneuvers, dynamic instabilities, and wind effects (gusts become a larger fraction of the freestream velocity) mean that aero/RCS interactions remain a concern at supersonic speeds.

Just before parachute deploy, six Entry Balance Mass Devices are jettisoned from the EV to move the $\mathrm{cg}$ back the the centerline. This puts the EV at a small angle-of-attack for safe parachute deployment. The aerodynamics of the EBMDs departing the vehicle are a concern in terms of recontact and possible aero interactions on the EV.

\section{Parachute Deploy and Heatshield Jettison}

The parachute deploys nominally near Mach 2.0. The parachute aerodynamics are beyond the scope of this paper. However, RCS use under the parachute may be performed to damp out rates introduced by the initial loads as the parachute opens as well as any excitations produced by the interaction of the capsule-parachute system.

Near Mach 0.8, the heatshield is jettisoned. The Viking project ran an extensive wind tunnel test to characterize the heatshield deploy envelope. ${ }^{1}$ It was decided to use this heritage data and stay within the defined envelope, rather than push towards a higher Mach heatshield jettison. The benefits to timeline did not justify the costs of a new experimental program and the accompanying CFD analysis. In this area, we claim Viking heritage and are bounded by that data.

\section{Powered Descent and Touchdown}

The rover and propulsion module stack, referred to as the powered descent vehicle (PDV), jettisons from the backshell following heatshield separation. The engines quickly ramp up and dominate the forces acting on the vehicle over any other aerodynamic forces. As the PDV approaches the ground, the rover is lowered on three tethers, while the descent stage slows the combined vehicle and then hovers as the rover touches down on the ground. This system is designed to keep the nozzle plumes away from the rover and far from the ground to minimize plume/ground interactions. However, this landing system is very different from the Viking lander which carried its descent engines under the lander deck. The threat of dust and sand to the rover (direct impact and deposition on the Rover deck) due to plume/ground interactions is one final issue where the Viking knowledge base is not applicable. This area is being in investigated by the MSL project with computational and experimental studies. However, the plume/ground interaction work is beyond the scope of this paper. 


\section{Analysis Tools}

A combination of several CFD codes, wind tunnel testing and engineering analysis was employed in the generation of aerodynamic data for the flight database and the focused assessments of the aerodynamic challenges specific to the MSL EV. The following briefly describes the important codes and experimental facilities used to support the findings reported in this paper.

\section{LAURA}

The MSL aerodynamics in the continuum regime in the aerodynamic database are generated with the Langley Aerothermodynamic Upwind Relaxation Algorithm (LAURA) ${ }^{2}$ CFD code. LAURA is a finite volume, shock capturing algorithm capable of calculating flows in chemical and thermal non-equilibrium. LAURA can be used for inviscid, thin-layer Navier-Stokes, or full Navier-Stokes computations. The calculations were performed on Intel Linux clusters (32-bit word length) with LAURA version "LAURA_intel" (last modified March 17, 2006). The calculations for a given solution were spread over many processors via Message Passing Interface (MPI). ${ }^{3}$

\section{OVERFLOW}

The NASA OVERFLOW 2 CFD code $^{4-6}$ was used to provide an independent assessment of supersonic static aerodynamics as well as to simulate the dynamic jettison of the balance mass devices prior to parachute deploy. OVERFLOW 2 is a CFD flow solver which simulates the Reynolds-averaged Navier-Stokes equations. These equations include the effects of compressibility and viscosity in the flow. The code can simulate a perfect gas or mixture of perfect gases. It uses overset structured grids to resolve the volume surrounding the aerodynamic body, with interpolation of flow quantities at computational boundaries to communicate between neighboring component grids. Simulations can be performed for steady or unsteady flows, and prescribed or 6-degree-of-freedom body motion can be included.

\section{Langley Unitary Plan Wind Tunnel (UPWT)}

The LaRC UPWT was used to measure the large angle-of-attack characteristics of the MSL EV at supersonic conditions. An early cold-jet RCS test was also conducted in UPWT to measure supersonic aero/RCS interactions. This continuous-running, closed loop facility has two test section legs, both with 4'x4' cross sections. The low-speed leg operates from Mach 1.5 to 2.86 and the higher speed leg can test from Mach 2.3 to 4.63. The MSL EV was tested at a Reynolds number of 2.0 million, which is close to flight conditions at supersonic speeds. As the MSL test reports are pending, Murphy et $\mathrm{al}^{7}$ provides an excellent description of the test facility and its use for a similar static force and moment test for the Orion command module.

\section{Langley 31" Mach 10 Hypersonic Tunnel}

The hypersonic aero/RCS interaction experimental program is currently between test phases in the 31" Mach 10 facility. ${ }^{8}$ This blow-down tunnel preheats the flow upstream of the nozzle. A heater coil and a highpressure air storage system provide plenum temperature and pressure of $1802 R$ and 1302 psi respectively. The air is expanded through a square, three-dimensional water-cooled nozzle with a $1.07 \mathrm{in}^{2}$ throat and $31 \times 31 \mathrm{in}^{2}$ exit area. The test section provides freestream dynamic pressure of $2.197 \mathrm{psi}$ and a unit Reynolds number of $1.798 \cdot 10^{6} \mathrm{ft}^{-1}$ at Mach 9.93. Models are injected into the tunnel through the side of the test section after start up and retracted prior to shut down to avoid excessive loads on the model. The total runtime of the tunnel is up to two minutes, although testing has shown that thermal effects prevent testing the MSL model for full-length runtimes. This facility and perhaps another Mach 10 facility at the Arnold Engineering Development Center (AEDC) are the highest Mach number test sections currently operating that are suitable for hypersonic force and moment testing. 


\section{Aerodynamic Challenges}

This section lists the important aerodynamic challenges identified and analyzed by the MSL project. The current assessment findings to date are presented and the objectives of ongoing work are described. While not an exhaustive list, the following shows the scope of problems that must be investigated by highlighting the important and novel challenges.

\section{A. Moderate Lift-To-Drag Flight}

\section{Hypersonic aerodynamics}

The Viking entry vehicle was designed to fly hypersonically at an L/D of 0.18 . MSL will be flying at an increased L/D of 0.24. This increased L/D is achieved with a greater cg offset but very similar forebody. Refer back to Figure 1 to see the geometry and cg differences between Viking and MSL. Wind tunnel based pitching moment curves, determined with three different freestream gases, intersected at the Viking trim angle of $11.2^{\circ}$ and $\mathrm{L} / \mathrm{D}$ of $0.18 .^{9}$ Historical literature does not explicitly state that the Viking hypersonic trim angle of attack was selected because identical trim point in hypersonic tests using three different gases. However, it is stated that the Viking project did not anticipate any significant deviations from their expected performance, using the intersecting pitching moment curves as justification.

At the time the Viking EV aerodatabase was being developed, CFD was in its infancy and no codes existed that could validate the hypersonic wind tunnel data. For the Mars Science Laboratory EV, CFD codes have replicated the Viking experimental data, showing the same intersection of $\mathrm{Cm}$ curves for different gases as well as the areas where the Cm curves diverge. LAURA CFD also closely replicates the reconstructed Viking angle-of-attack history. Figure 3 shows the reconstructed angle-of-attack and L/D history determined from instrumentation on board the Viking EV compared with a "prediction" determined with MSL aerodatabase (CFD) data transferred to the Viking cg location.

LAURA CFD also captured bounded instabilities caused by real gas effects in $\mathrm{CO}_{2}$ for the Mars Pathfinder, Mars Exploration Rover and Mars Phoenix entries. ${ }^{10-12}$ Therefore the MSL project has reasonable confidence that computational capabilities can effectively predict hypersonic trim characteristics in $\mathrm{CO}_{2}$ and can therefore fly with a L/D and trim angle up to those flown by Viking. Even with slight differences in shoulder radius, trim angle is in agreement within about $0.5^{\circ}$. However, flying at even greater angles with a larger vehicle introduces new mechanisms that affect the predicted trim angle which must be accounted for properly.

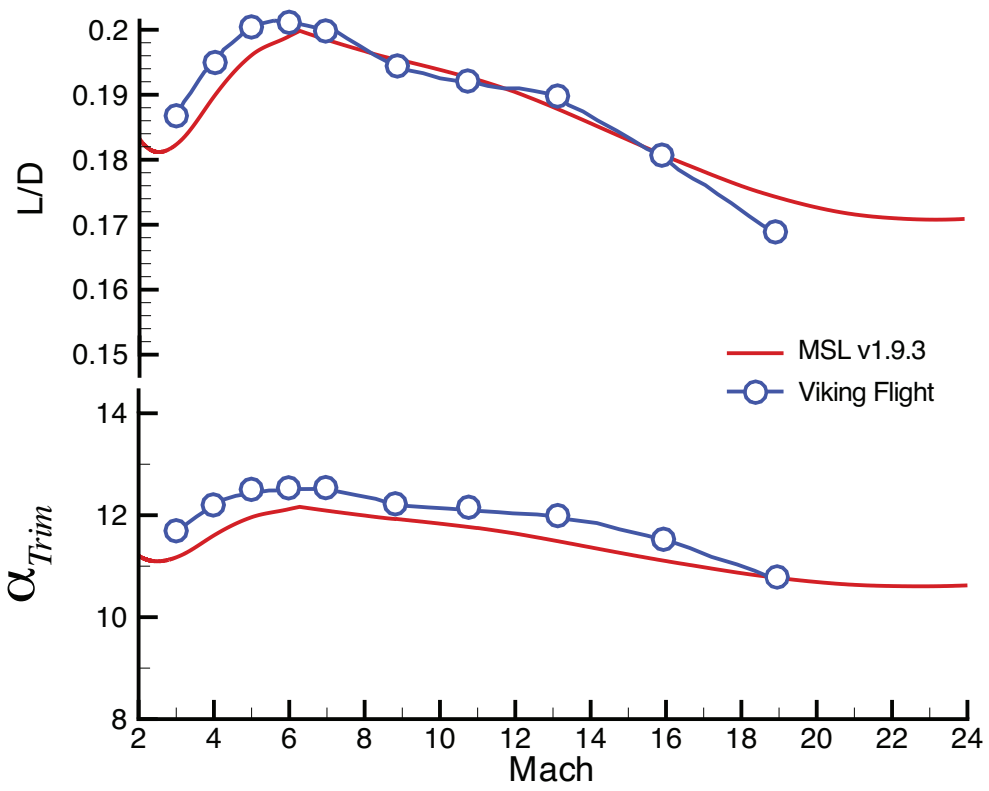

Figure 3. Comparison of MSL "predictions" to reconstructed Viking flight data 
Figure 4 shows two sets of CFD predictions, laminar and turbulent, run at $5200 \mathrm{~m} / \mathrm{s}$ and $2400 \mathrm{~m} / \mathrm{s}$. These cases (and others at intermediate velocities) have shown that turbulence can have an effect on trim angle. Three potential mechanisms are thought to be contributing to these differences. First, the fully turbulent boundary layer has greater shear stress acting on the lee-ward side that adds an additional pitching moment increment. Also, an increased boundary layer thickness corresponds to a thicker displacement thickness that causes the lee-side of the EV forebody to act like a more blunt body, also producing a pitching moment delta. Finally the turbulent cases show the shock wave is closer to the forebody than the laminar case. This is an indication that the global pressure change across the shock is different between the laminar and turbulent cases. Relative contributions to the pitching moment from each of these effects are being investigated currently. Verification of CFD results is ongoing to ensure that the effects predicted will be seen in flight.
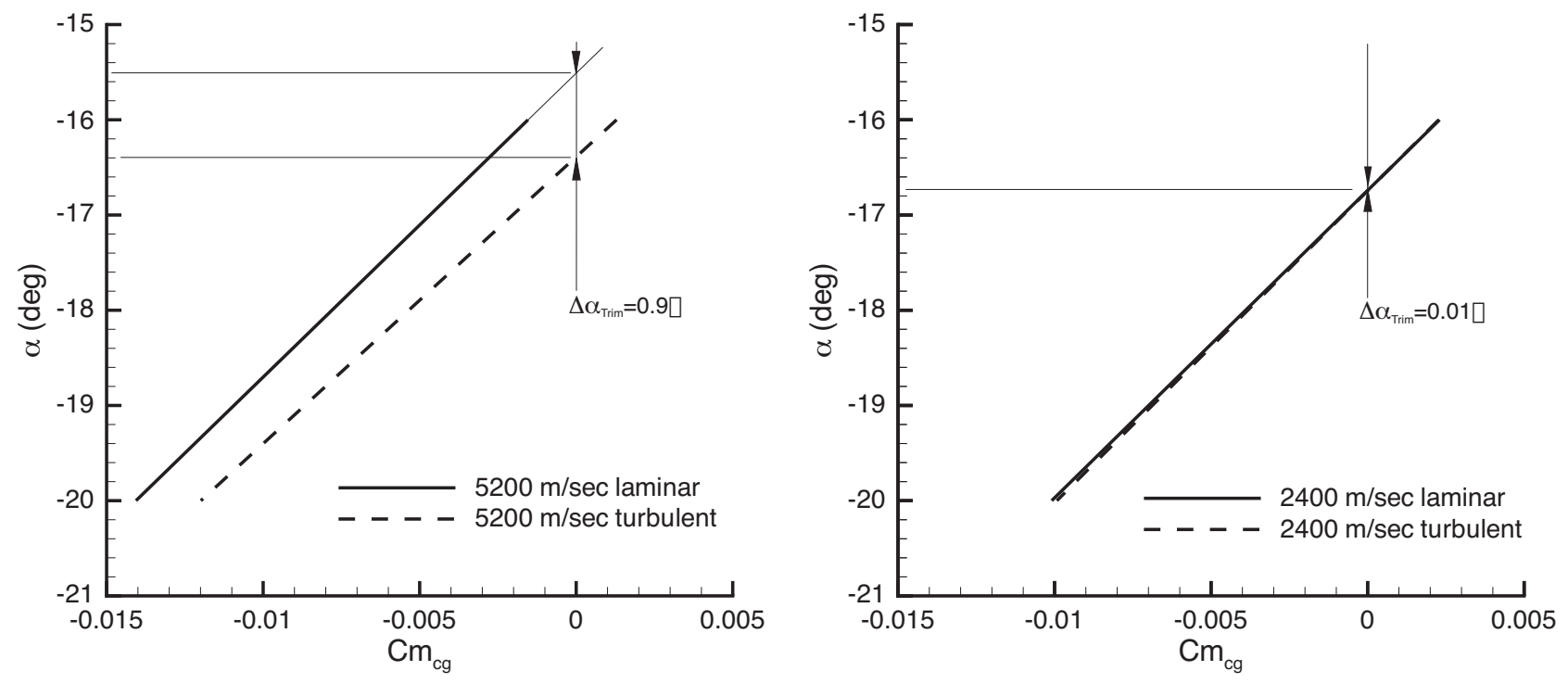

Figure 4. Hypersonic turbulent and laminar pitching moment predictions

The question of force and moment differences due to laminar or turbulent flow on the forebody has been assessed for previous Mars missions with supersonic wind tunnel data and CFD calculations (usually at small angles-of-attack and at lower speeds then the problem-cases identified for MSL). In general, all prior data has shown that laminar and turbulent aerodynamic forces and moments are essentially the same. The mechanisms identified appear to be valid at all angles at hypersonic speeds. However, differences between leeward and windward flowfields increase with angle-of-attack, as the stagnation point moves to one side of the vehicle and the flow running length becomes much greater on the leeward side. This is an area of ongoing investigation for the MSL project. The effects of some mechanisms might be unrealistically amplified by the specification of all-laminar or all-turbulent conditions in the CFD solutions. This is an area of the MSL flight envelope where exceeding that of Viking may be introducing constraints on how CFD is computed to accurately predict trim angle. The correct modeling of laminar and turbulent regions on the forebody might be important to resolve the trim angle at high hypersonic velocities at trim angles greater than Viking. CFD analysis has not been used on any Mars mission that has flown at significant angle-of-attack and therefore did not identify this issue.

In addition to questions of laminar or turbulent flow, LAURA CFD solutions have also shown sensitivities to trim angle due to gas chemistry models at some hypersonic Mach numbers. LAURA CFD predictions showed a sensitivity of trim angle-of-attack to $\mathrm{CO}_{2}$ temperature relaxation rates used in the non-equilibrium solutions at hypersonic speeds. The models by Camac ${ }^{13}$ and Millican and White ${ }^{14}$ were used in LAURA solutions and compared at points along the entry trajectory. The largest differences were found near Mach 9. Figure 5 shows two pitching moment curves generated with CFD predictions using the two relaxation rates.

This comparison and the sensitivities shown in Figure 4 show that for the Viking shape flying at angle- 


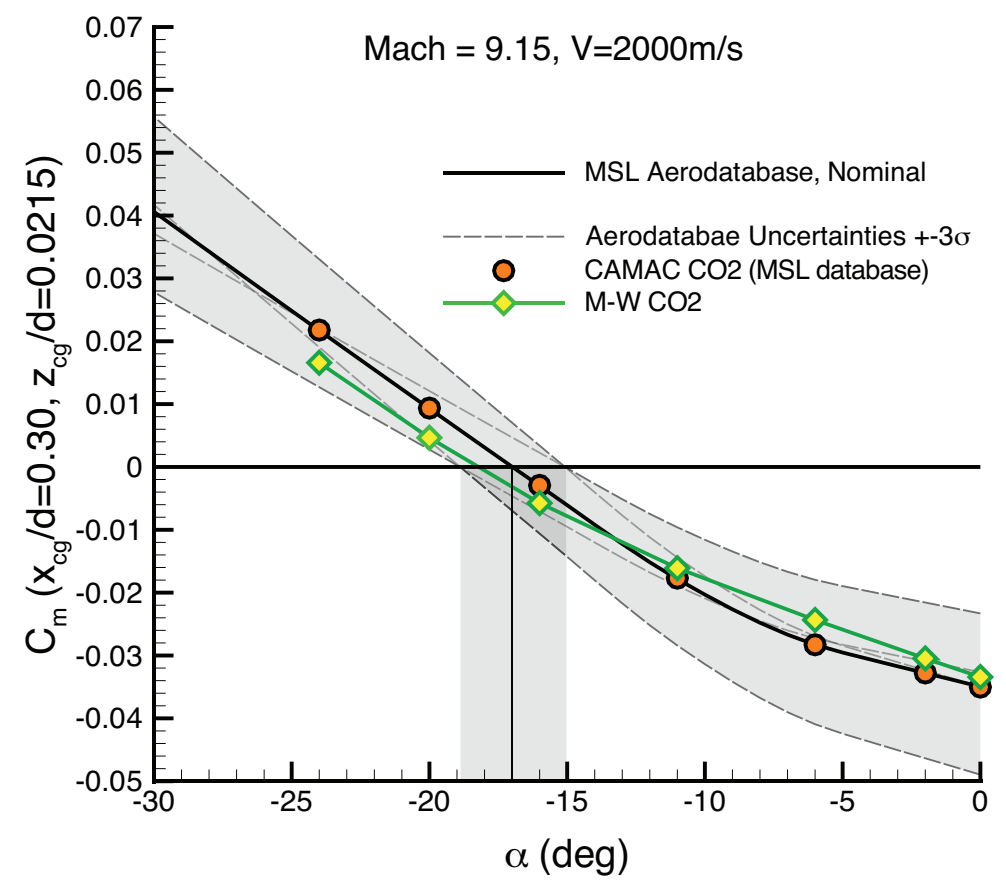

Figure 5. Hypersonic $C_{m}$ sensitivities due to LAURA temperature relaxation rate models

of-attack, there are many more chemistry and aerodynamic flowfield mechanisms that can affect the pitching moment and trim angle-of-attack. When flying ballistically, the EV benefits from symmetry and will always have a trim point at $\alpha=0^{\circ}$. A detailed analysis of all these sensitivities and their variation with trim angle, Mach number (or velocity), and density is being conducted and will be synthesized into a comprehensive hypersonic uncertainty model, and possibly some changes to the models and flow conditions in the nominal CFD solutions. Pitching moment uncertainty has been increased from that used in recent ballistic entry Mars missions. The uncertainty model will be detailed below. The MSL project is currently working to develop procedures to systematically disperse chemical reaction rates and other gas chemistry parameters within the LAURA code to more rigorously quantify uncertainties on hypersonic CFD predictions.

\section{Supersonic aerodynamics}

The claim of Viking heritage comes under question at supersonic speeds as well. The cg offset that provides the desired hypersonic L/D becomes more effective at supersonic speeds. Figure 6 shows the predicted L/D and $\alpha_{\text {Trim }}$ for the MSL EV. Just before the balance mass jettisons ( Mach 2.5), the capsule trims at approximately $-20^{\circ}$. The Viking aerodatabase data only goes up to $23^{\circ}$. As the MSL capsule oscillates in flight it is possible that it might experience angles of attack exceeding any relevant historical data. Furthermore, the backshell contribution becomes a significant contributor to the drag acting on the vehicle at supersonic speeds. At large angles of attack, the flow can become attached and alter the pitching moment. All recent Mars missions used forebody CFD solutions with a Viking-derived base pressure correction for the axial force coefficient. ${ }^{11}$ It was not known if this approach was still valid at large angles-of-attack.

As the MSL backshell has a different outer mold line (OML) than any previous and the trim angle-ofattack is to be greater than any Mars mission flown, it was decided that supersonic validation wind tunnel data should be obtained to quantify any additional influence on aerodynamic performance due to backshell contributions. For controller design and the accurate simulation of entry flight performance, it is important to determine if the static pitching moments become nonlinear at larger angles of attack. This becomes more likely at large angles when flow is attached on portions of the backshell and separated on others. LAURA CFD is notoriously poor at predicting even the absolute average wake pressure acting on a backshell, and equally poor at determining attached and separated regions of the flowfield. 


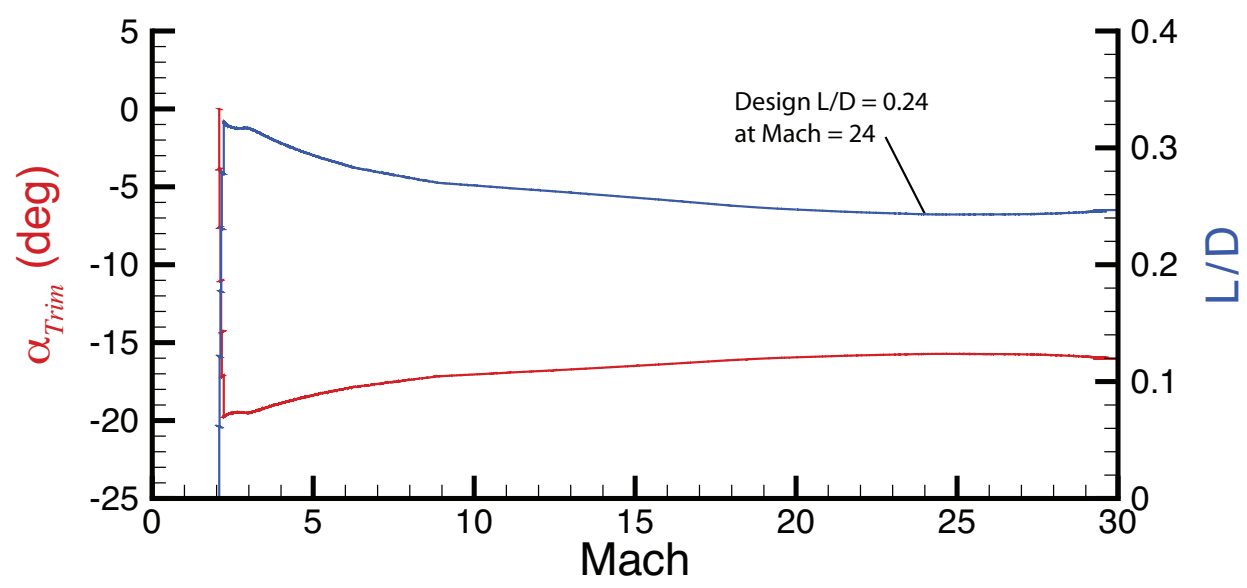

Figure 6. Predicted L/D and trim angle-of-attack for Mars Science Laboratory entry vehicle

Force and moment testing was conducted in the NASA Langley Unitary Plan Wind Tunnel (UPWT). This facility has two legs which can test at Mach numbers from 1.2 to 4.5. Two different sting angles were used to minimize their effects at different angles of attack. Comparisons of data taken at the same model angles-of-attack helped to assess influence of the sting on the forces and moments acting on the model.

Comparisons presented here are made against the UPWT $30^{\circ}$ sting data. This data has smaller sting effects near trim angle-of-attack $\left(\alpha_{\text {Trim }} \approx-20^{\circ}\right)$ across the Mach range presented. Figures 7 and 8 show comparisons of axial force and pitching moment coefficients. The LAURA CFD-based aerodatabase data are plotted with $\pm 3 \sigma$ dispersion bounds. The uncertainty bounds are defined in a later section.

In general there is good agreement between the data, independent of the particulars of the vehicle (Viking or MSL), turbulence model, or environment (wind tunnel or Mars flight conditions). The OVERFLOW CFD solutions are plotted as representative cases from a much larger set of independent computations where turbulence models (including laminar solutions) and sting configuration were varied for three different CFD codes (OVERFLOW, FUN3D ${ }^{15}$ and LAURA). The deviation of the OVERFLOW data shown here is typical of deviations from experimental data for all codes. Specific trends could be attributed to running cases with laminar flow or any turbulence model. No particular combination of CFD code and turbulence model could be rigorously defended as "correct," but together gave an idea of the sensitivity of the data to the various parameters. The comparisons provided no evidence that uncertainties should be increased. Conversely, the variation due to turbulence models and CFD codes confirms there is no justification in reducing the uncertainties.

The comparison plots show the large angle data obtained in the Langley UPWT facility up to a total angle of $36^{\circ}\left(\alpha=-36^{\circ}\right.$ in the MSL coordinate system). The CFD-derived aerodatabase, comparative CFD solutions, and Viking data all generally agree with the UPWT trends at their respective angle-ofattack limits. No significant nonlinearities or dramatic changes in the character of the static aerodynamic curves were observed in the supersonic wind tunnel test data. Based on these comparisons, any additional uncertainties introduced by MSL flying at a greater supersonic L/D than Viking are captured in the current aerodynamic dispersions. The conclusion from this test data is that the effects of the MSL backshell on the static aerodynamics are small up to large angles of attack and so the methods used for recent Mars missions remain valid.

\section{B. RCS/Aerodynamics interactions}

The area that has demanded the most attention of the MSL aerodynamics team is that of aero/RCS interactions. The RCS jet positions and orientation were driven by mechanical, aerodynamic, and aero-heating considerations. The EV mechanical designers suggested several preliminary configurations. Each design iteration required analysis by the aerodynamics and aerothermodynamics teams. Each of the early versions was shown to be unacceptable for a reason related to aerodynamics (large aerodynamic interactions) or 

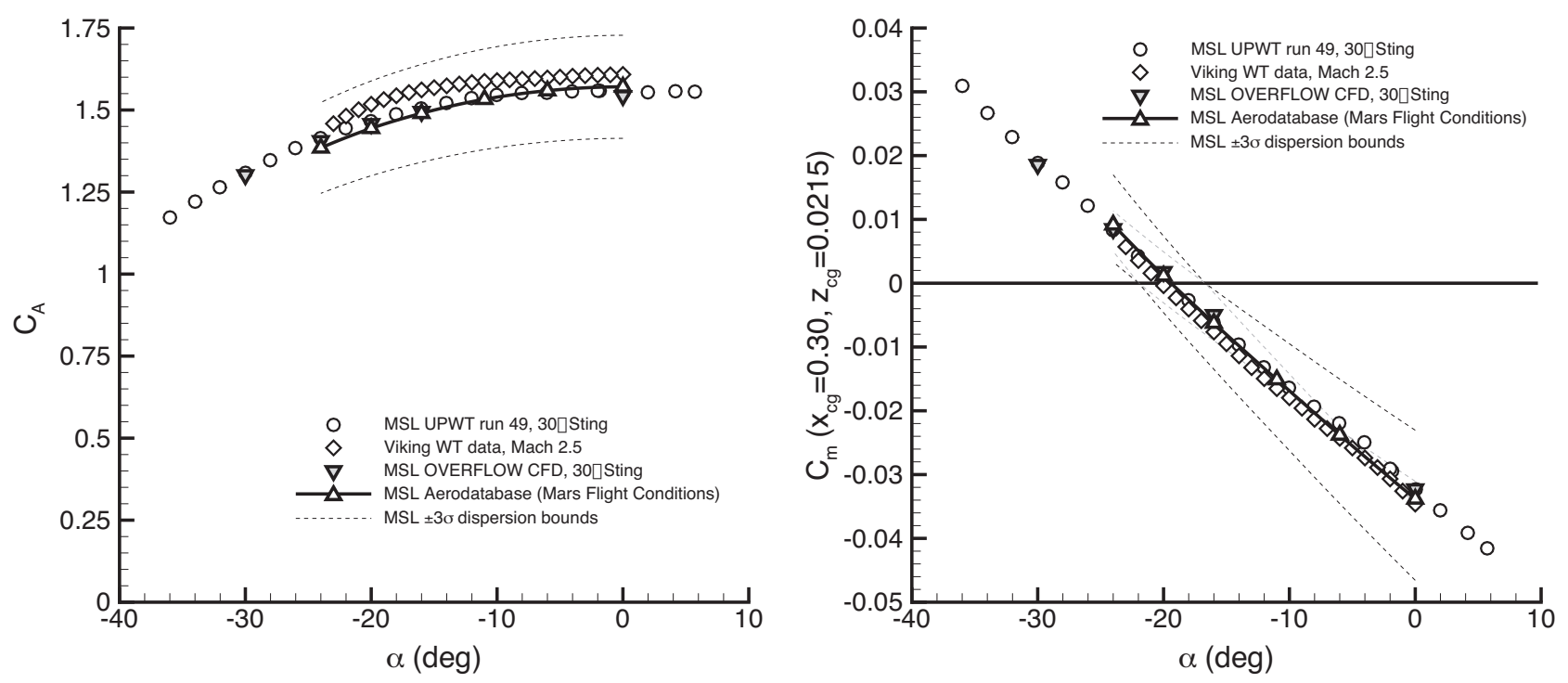

Figure 7. Comparison of static aerodynamic data sources, Mach 2.5
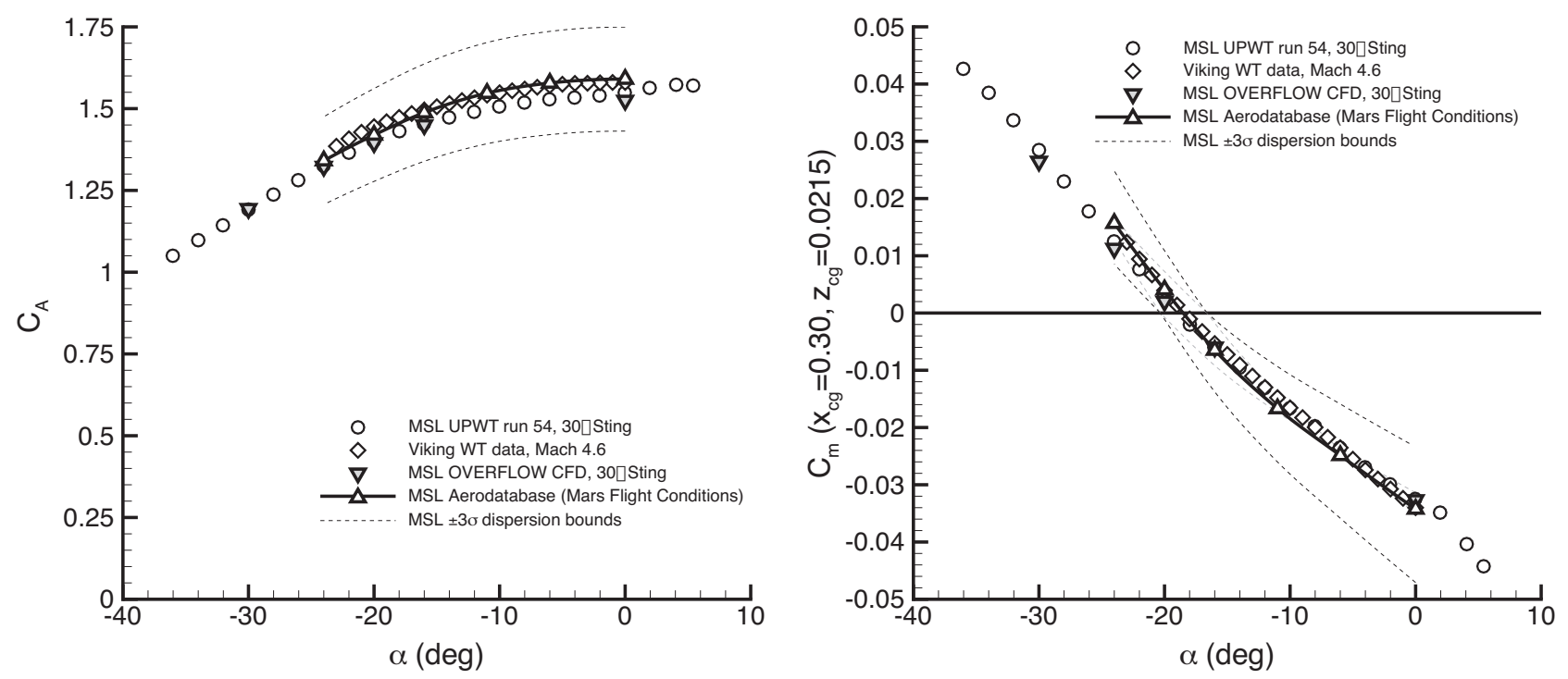

Figure 8. Comparison of static aerodynamic data sources, Mach 4.5 (unless specified) 
aerothermodynamic heating (excessive heating of the jets or the backshell environment in their vicinity). The details of earlier configurations are beyond the scope of this paper. The final configuration is in the process of final validation wind tunnel testing at the time of writing. This section will give a brief summary of the design philosophy that led to the final configuration and the CFD analysis and wind tunnel testing that is tasked with quantifying the aero/RCS interactions. The final aero/RCS interaction model has not been completed, so no specifics will be presented.

\section{Design Considerations}

Two papers by Dyakonov et $\mathrm{al}^{16,17}$ provide excellent overviews of the design considerations that have been identified as important for minimizing aero/RCS interactions for the MSL EV along with a good comparison with previous RCS-stabilized vehicles that have flown to Mars.

Due to payload design and packaging constraints, the MSL RCS configuration differs from the Viking configuration. Figure 9 shows the Viking and MSL RCS configurations. Viking had independent roll, pitch, and yaw jets made of a combination of 12 jets (eight produce pitch or yaw, four produce pure roll). MSL uses four pairs (eight total) of jets canted so as to provide roll pitch and yaw components from each jet. Roll, pitch and yaw control are achieved by selecting the proper pairs.

The RCS jets use the same mono-propellant (hydrazine) as the landing engines. There was insufficient clearance or mass margin to place the RCS engines out near the maximum diameter and it was decided to mount the jets on the powered descent vehicle to eliminate the complexity of separating the jets from the payload when the lander drops out of the backshell to begin powered descent. This mechanical accommodation reduced the moment arms about each axis, but the jets were sized to provide sufficient angular accelerations for robust control of the EV during entry. With the change in RCS configuration and changes to the backshell shape, Viking heritage was lost and the RCS interactions must be quantified to ensure that control laws are properly defined.

The RCS design iterations resulted in what is believed to be the best combination of control authority and minimization of aero/RCS interactions given the available RCS location space as constrained by the lander mechanical systems. This belief is supported by CFD analysis and ongoing experimental data. All analysis and experimental data to date shows that the flight configuration has healthy control authority with small cross interactions, although this work is ongoing.
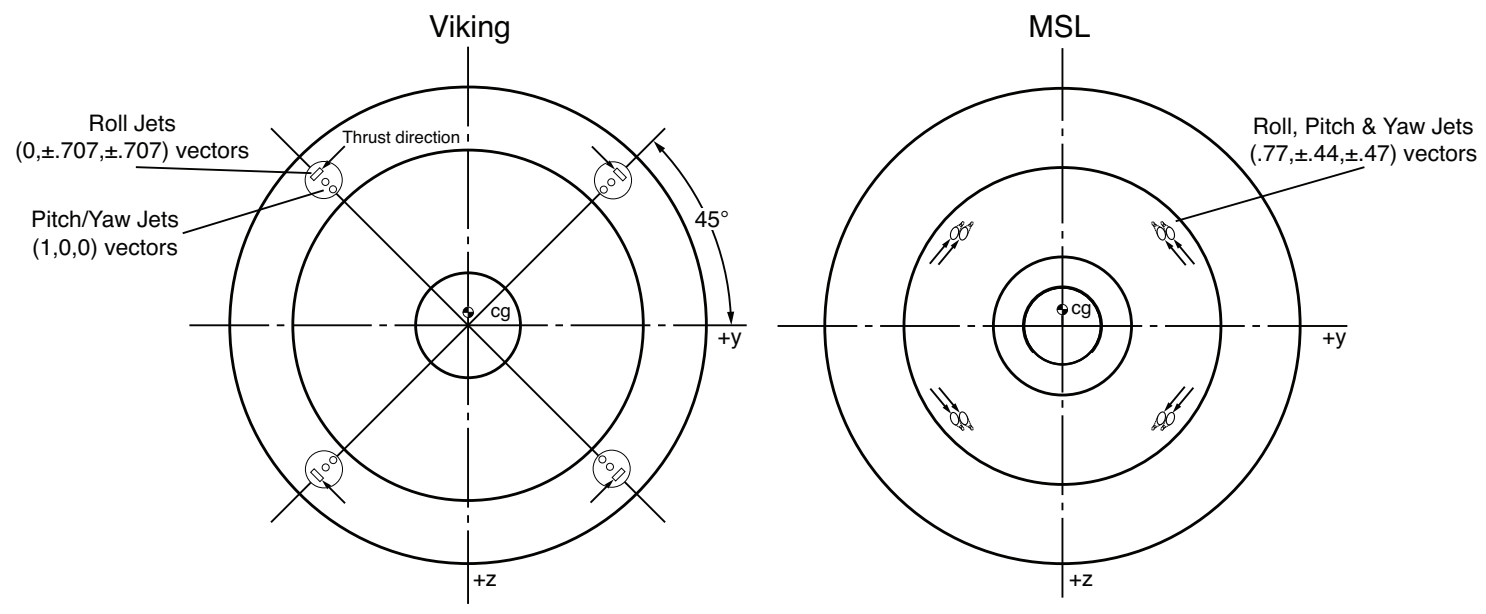

Figure 9. Comparison of MSL and Viking RCS jet location and orientations (looking at backshell)

During the design process it was determined that the hypersonic segment of the entry profile near peak dynamic pressure is the regime most conducive to large aero/RCS interactions. This was not immediately recognized, but discovered when an early hypersonic CFD solution of a preliminary RCS configuration showed complete negation of the yaw authority. This finding resulted in the redesign of the RCS configuration to its 
current state. ${ }^{16}$ Looking into the cause of the large interaction revealed a plausible explanation, supported by Viking flight data, for why hypersonic conditions can produce the largest interactions.

The Viking entry vehicles each carried a single pressure tap on the backshell to measure wake pressure during entry. As expected, at hypersonic speeds, the wake pressure was a negligible fraction of the forebody stagnation pressure. At supersonic speeds, the pressure becomes a sizeable contributor to the EV drag. This measured pressure variation was applied as a $C_{A}$ correction to forebody-only CFD solutions for all recent Mars missions starting with MPF. Mitcheltree developed a curve fit base correction anchored to Viking flight data and some CFD calculations. ${ }^{11}$ In terms of axial force coefficient corrections, the base contribution at hypersonic conditions is very small. However, the absolute pressure in the wake actually peaks near peak dynamic pressure (Figure 10). These peak pressures happen to be a tiny fraction of the forebody pressures and need not be accounted for in the static aerodynamic coefficients. However, the RCS moments are also a tiny fraction of the aerodynamic moments at hypersonic conditions. The backshell pressures are large enough negate the intended RCS torques if disrupted by the RCS plumes in the right way.

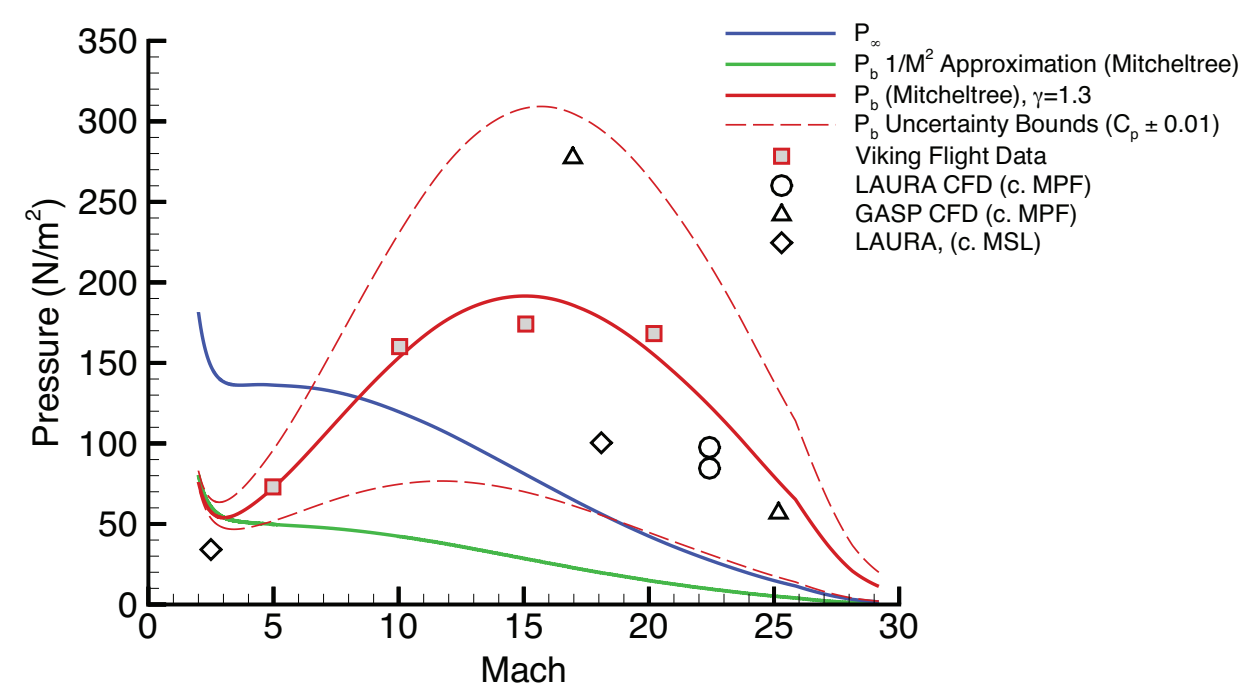

Figure 10. Viking-based backshell pressure model applied to MSL nominal trajectory

\section{Computational analysis}

Several computational assets were applied to the aero/RCS investigation. CFD predictions of the RCS influence on the EV aerodynamics at Mars flight conditions as well as wind tunnel conditions (with and without the model sting) were calculated using LAURA and FUN3D CFD codes. As an evaluation tool, the CFD solutions were useful in visualizing wake flowfields and identifying some significant pressure changes on the backshell induced by the RCS plumes.

At several points along the entry trajectory, CFD was used to evaluate a "box" of orientations circumscribing the trim angle of attack: $\alpha_{\text {Trim }}, \beta_{\text {Trim }}$ and the maximum and minimum $\alpha, \beta$ and $\alpha / \beta$ attitudes expected in flight. At each orientation of the box, calculations of the aero/RCS interactions were calculated for each combination of thruster firings (roll, pitch, yaw jet combinations and individual jet-pairs) to look for any significant aero/RCS interactions the vehicle might encounter within its expected range of attitudes during entry. The bounding box was typically defined at $\pm 10^{\circ}$ in $\alpha$ and $\beta$ from the nominal trim angle.

Figure 11 shows examples of two LAURA solutions demonstrating the mitigation of the yaw RCS interactions by changing to the final RCS configuration. Details of the CFD analysis can be found in the papers by Dyakonov et al. 

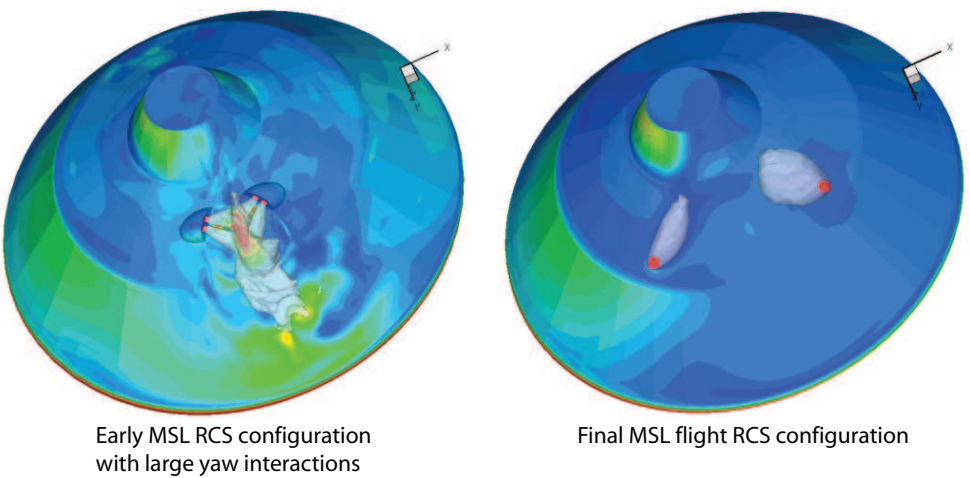

Figure 11. Example CFD solutions showing effects of RCS jet location on backshell interactions (LAURA CFD at Mach 18.1) 16

CFD was also used to predict the matrix points of the planned supersonic and hypersonic wind tunnel tests. The solutions were computed prior to testing in order to determine CFD's accuracy as a predictive tool for RCS interactions. The blind prediction and the RCS data forms the final information which will determine the magnitude of the aero/RCS interaction dispersion model in the aerodatabase.

\section{Experimental measurements}

The initial intent of the MSL aero/RCS experimental test program was to validate the findings of preliminary CFD analysis. Validation in this context means experimental force and moment tests that show similar magnitudes of aero/RCS interactions. If the wind tunnel data showed that CFD predictions were not validated (complete disagreement showing serious deficiencies in the ability of CFD to estimate interaction effects), the wind tunnel data would be used to build conservative bounding aero/RCS interaction model.

A supersonic scale test of an early MSL OML and RCS configuration was conducted in the Langley UPWT in 2006. Figure 12 shows the model geometry and RCS jet layout for that test. While not matching the final configuration, it was determined that these jets would produce interactions similar to those of the final configuration. CFD analysis of both configurations at wind tunnel and flight conditions supported this conclusion.
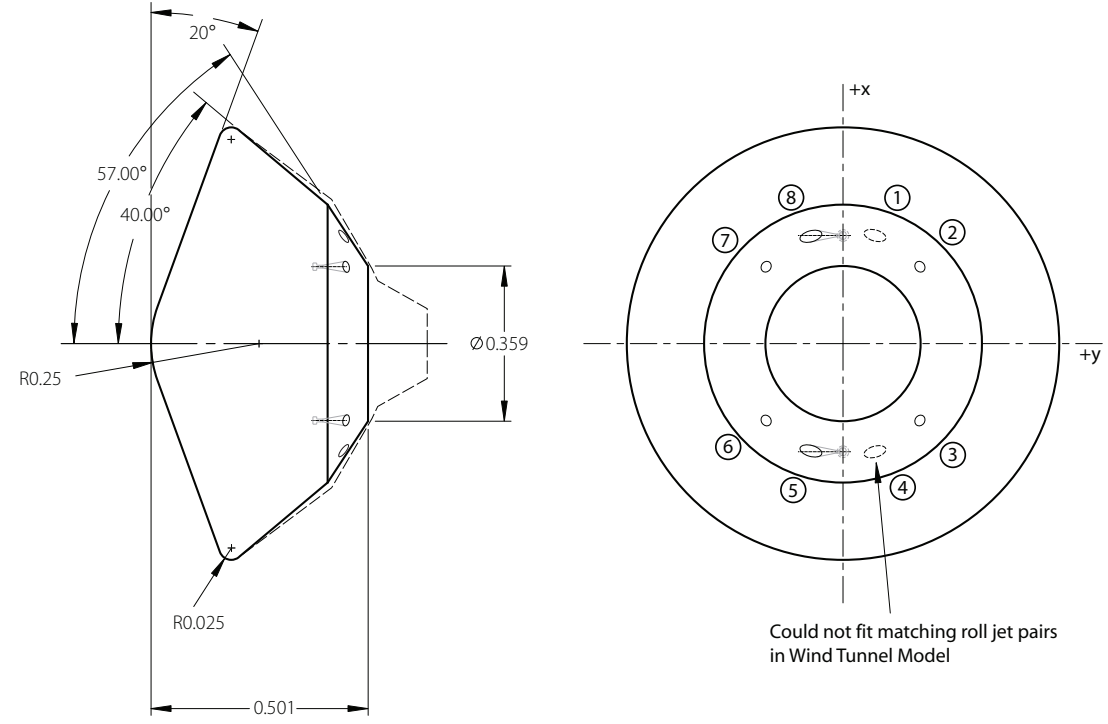

Figure 12. OML6 RCS configuration as tested in LaRC UPWT, Dimensions normalized by model diameter $\left(d_{\text {model }}=4.5 \mathrm{in.}\right)$ 
A model of the MSL OML and final RCS configuration is between test phases in the Langley 31" Mach 10 Hypersonic Tunnel at the time of this conference. Figure 13 shows the Mach 10 model. A preliminary test phase determined the best practices to obtain reliable aerodynamic interaction increments due to the RCS jets. Several thermal effects and balance loading problems were encountered and testing is on hiatus as the issues identified in phase I are addressed.

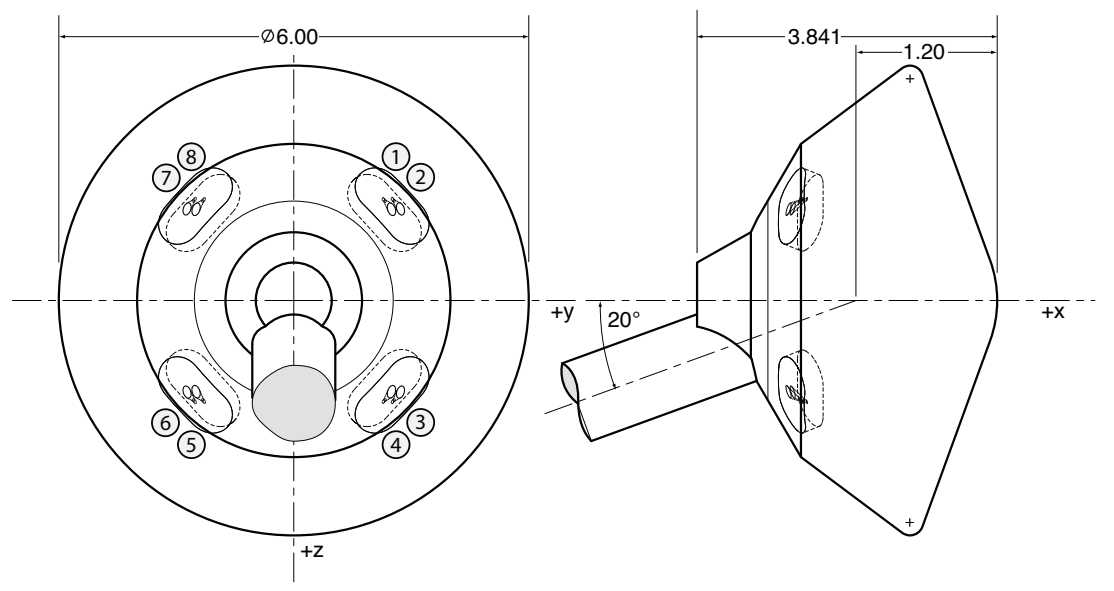

Figure 13. OML 13F Option-M RCS configuration as tested in LaRC 31" Mach 10 tunnel, dimensions in inches

Based on previous RCS test experiences with the Shuttle Orbiter, ${ }^{18}$ the RCS jet plumes were approximated with conical nozzles with a lower exit Mach number, but higher mass flow. The nozzles were sized to match the ratio of jet momentum to freestream momentum. This was found to be the most important scaling parameter for RCS scale testing of the Orbiter. The nozzle cone angles were selected to produce a plume shape matching the full scale plumes using a code developed by Salas. ${ }^{19}$

Equating the flight and scale model momentum ratios, the chamber pressure required can be determined for a given scale model and nozzle shape.

$$
P_{c, \text { tunnel }}=\left(\frac{\gamma_{e}}{\gamma_{\infty}} \frac{P_{e}}{P_{c}} \frac{A_{e}}{S} \frac{M_{e}^{2}}{M_{\infty}^{2}}\right)_{\text {flight }}\left(\frac{\gamma_{\infty}}{\gamma_{e}} \frac{P_{c}}{P_{e}} \frac{S}{A_{e}} \frac{M_{\infty}^{2}}{M_{e}^{2}}\right)_{\text {tunnel }} P_{c, \text { flight }} \frac{P_{\infty, \text { tunnel }}}{P_{\infty, \text { flight }}}
$$

where

$$
\frac{P_{c}}{P_{e}}=\left(1+\frac{\gamma-1}{2} M_{e}^{2}\right)^{\frac{\gamma}{\gamma-1}}
$$

Table 1 shows the flight conditions at Mach 10 and the scaled values for those parameters required to match the momentum ratio in the Mach 10 tunnel. Nozzles for the earlier supersonic model were designed using the same scaling relations.

Force and moment testing in a hypersonic wind tunnel presents many additional challenges beyond scaling. Most have their root in the fundamental problem of trying to measure very small interactions on the backshell of the model using a balance that must be sized to withstand aerodynamic loads on the forebody. Preliminary test design showed that balance uncertainties would be approximately 10 to $25 \%$ of the RCS torques (matching the Mach 10 flight momentum ratio). The influenced the interpretation of test results. The test would look for aero interactions larger than the balance uncertainties. The measurement accuracy was not such that a high fidelity interaction model might be built. Rather, the test is looking for any large interactions that CFD did not predict, but should be accounted for in the flight interaction model. Preliminary measurements show that interactions appear to be small, but balance heating effects and other tunnel operational concerns must be addressed before the final data runs.

\section{Initial CFD/Experiment comparisons}

Figure 14 shows a representative comparison of UPWT test results with two CFD predictions. 
Table 1. 31" Mach 10 Nozzle Design Variables scaled to match flight

\begin{tabular}{ccc}
\hline Variable & Tunnel & Flight \\
\hline \hline$\gamma_{e}$ & 1.4 & 1.346 \\
$\gamma_{\infty}$ & 1.4 & 1.313 \\
$M_{e}$ & 3.51 & 4.47 \\
$P_{c}$ & 77.363 & 334.79 \\
$P_{e}$ & $3.644 \cdot 10^{-6}$ & 0.00329 \\
$A_{e}\left(m^{2}\right)$ & 0.01824 & 15.904 \\
$S\left(m^{2}\right)$ & 9.93 & 10.0 \\
$M_{\infty}$ & 220.32 & 137.72 \\
$P_{\infty}\left(N / m^{2}\right)$ & 0.550 & 289.3 \\
$P_{c}\left(N / m^{2}\right)$ & $6.766 \cdot 10^{5}(98.1 p s i)$ & $1.0935 \cdot 10^{6}(158.6 \mathrm{psi})$ \\
\hline \hline$(\dot{m} V)_{\text {jet }}(N)$ & 554.93 & $2.92033 \cdot 10^{5}$ \\
\hline \hline$(\dot{m} V)_{\infty}(N)$ & &
\end{tabular}

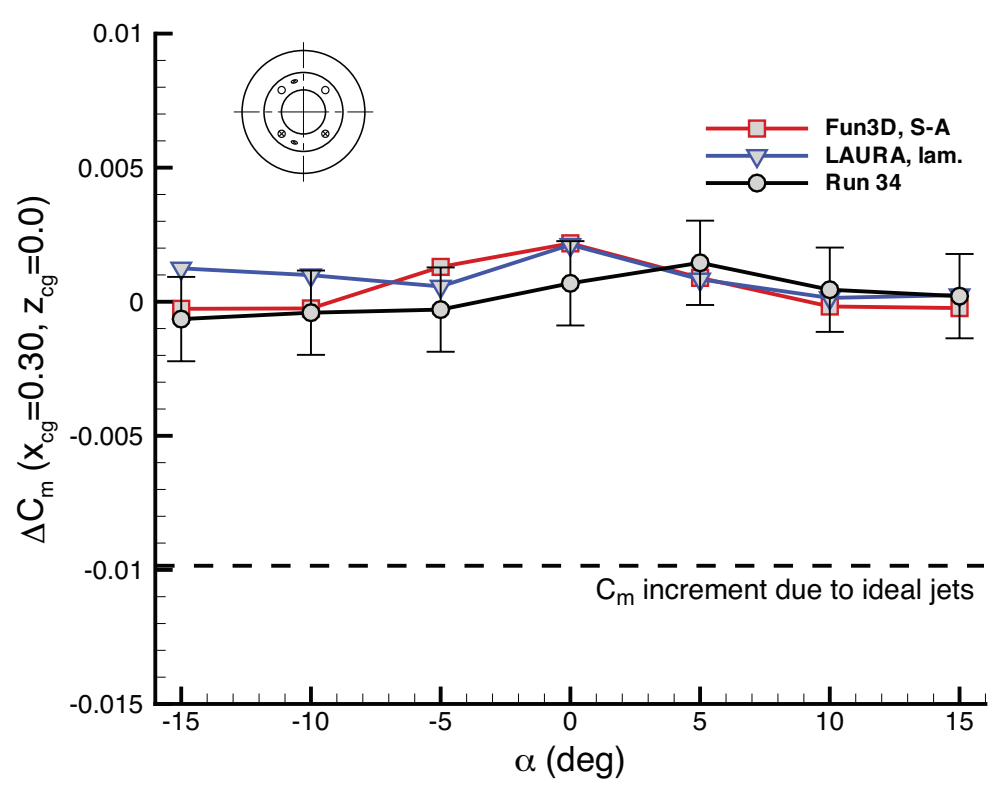

Figure 14. OML6 CFD/UPWT test comparison of aero/RCS interactions, Mach=2.5, $-C_{m}$ pitch jet pair 
The comparisons show that CFD generally captures the proper magnitude of interactions, but does not necessarily predict the direction/orientation of the resultant moment increment. At some angles of attack the two CFD codes predict increments of opposite sign. The aero RCS model will be defined by these comparisons. The general approach (completed at supersonic speeds, the Mach 10 analysis is ongoing) is to run CFD predictions of a full set of RCS firing combinations (roll, pitch and yaw) at a range of angles-ofattack and sideslip bounding those that will be seen at flight conditions (using multiple codes in some cases). The solutions show a range of interactions and the largest are noted. The experimental data then replicate the test points, looking for any data that suggests the CFD predictions have grossly missed the magnitude of the aero/RCS interactions at any particular orientation or jet combination. The supersonic interaction magnitudes were found to be in reasonable agreement. The interaction model is a conservative bound on the CFD and wind tunnel values, centered about a nominal of zero interactions. This approach is taken for two reasons. First, the accuracy of CFD has not been validated by wind tunnel data, so a high fidelity RCS interaction model would have to be built from extensive (and costly) wind tunnel testing. Second, the interactions are small. Because the RCS configuration has produced only small interactions compared to the nominal RCS torques, there is no need to build a higher fidelity model with detailed Mach and $\alpha, \beta$ functionality. Conservative interactions that bound all values measured do not break the controller logic. This approach has yet to be completed for the hypersonic regime, pending the completion of the Mach 10 test program. Also, further stress testing of the controller in 6-DoF simulation may reveal flight performance issues that might warrant a return to the supersonic tunnel with the final RCS configuration as well.

\section{Entry Balance Mass Jettison}

The MSL capsule flies at a lifting attitude $(\mathrm{L} / \mathrm{D}=0.24$ at Mach 24$)$ through most of entry, achieved with a center-of-gravity offset from the spin axis of symmetry. To safely deploy the parachute at Mach $=2.0$, it is necessary to have the capsule at a small angle of attack. The low-angle attitude ensures the parachute deploys in alignment with the velocity vector into the subsonic wake flow and will not see a significant crossflow component that could damage the parachute. MSL changes the trim angle-of-attack by jettisoning balance masses overboard, moving the cg onto the vehicle centerline. Six of tungsten slugs are jettisoned overboard in sequence, one every two seconds, starting when the EV slows to Mach 2.5 and finishing just before parachute deploy. Figure 15 shows a simplified diagram of an EBMD. The mass of each EBMD has increased slightly with design iterations and is currently allocated at $29 \mathrm{~kg}$.
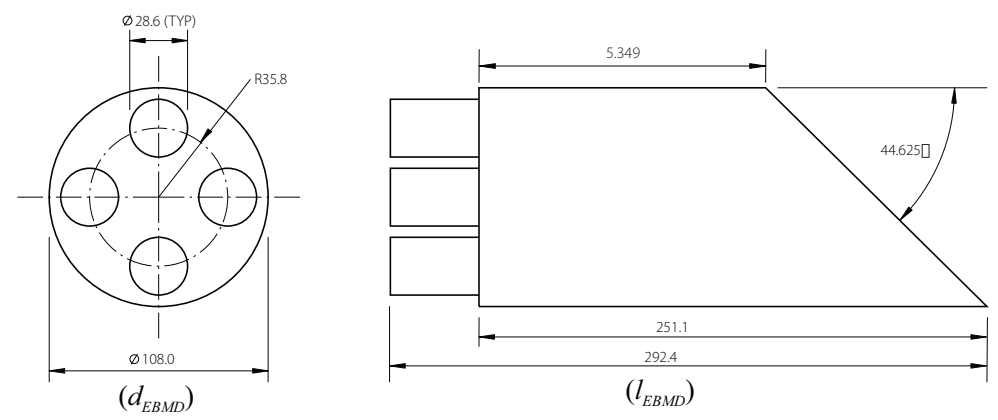

$S_{E B M D}=\left(v_{\text {EBMD }}\right)^{2 / 3}=\left(\pi / 4 d^{2} l\right)^{2 / 3}=0.0193 \mathrm{~m}^{2}=29.895 \mathrm{in}^{2}$

Figure 15. Simplified Entry Balance Mass Device geometry (dimensions in millimeters).

This mechanical system provided another new aerodynamic challenge for the Mars Science Laboratory entry vehicle. Will the jettisoned masses safely depart the vehicle with dynamic disruption or recontact with the EV? The ratio of ballistic coefficients shows that the balance mass will definitely separate from the capsule with no chance of long-term recontact. The ballistic coefficients differ by more than an order of magnitude (the values used in Equation 1 are representative but not exact as the balance mass and total EV mass have not finalized): 


$$
C_{B, E B M D} / C_{B, E V}=\frac{27.7 \mathrm{~kg}}{1.0 \cdot 0.0193 \mathrm{~m}^{2}} \frac{1.5 \cdot 15.9 \mathrm{~m}^{2}}{2900 \mathrm{~kg}}=11.8
$$

While long-term separation is assured, a near-term recontact possibility was identified. As the EBMDs are jettisoned they fall out past the vehicle shoulder through rapidly changing flow conditions before passing through the EV's bow shock. Higher fidelity analysis was done to assess the separation dynamics beyond the preliminary zeroth order analysis.

The springs that jettison the balance masses were sized to provide a $1 \mathrm{~m} / \mathrm{s}$ separation velocity in the direction collinear with the EBMD spin-axis. The separation velocity has been validated with ground-based mechanical testing. This relatively low velocity allows the chance that rates imparted on the EBMD might combine with EV oscillations at the time of jettison and result in recontact as the masses separate and pass round the EV shoulder. Design requirements demand that no recontact occur for any separation event. This obviates the need to imagine and assess all the different kinds of recontact and quantify the allowable energy of such events.

To analyze the near-body separation, two analyses were run. A first order planar simulation was run at different capsule angles of attack (approximating the trim angles as each of the six EBMDs are jettisoned) to assess the effects of flow density, dynamic pressure and angle of attack during the entire EBMD jettison window. Figure 16 shows the results of the planar analysis. The angle of attack had a small effect on the trajectory of the EBMD as it departed the EV, but overall the separation happens very quickly and consistently for the conditions evaluated. The initial separation velocity of $1 \mathrm{~m} / \mathrm{s}$ appears to be sufficient in this first order analysis.

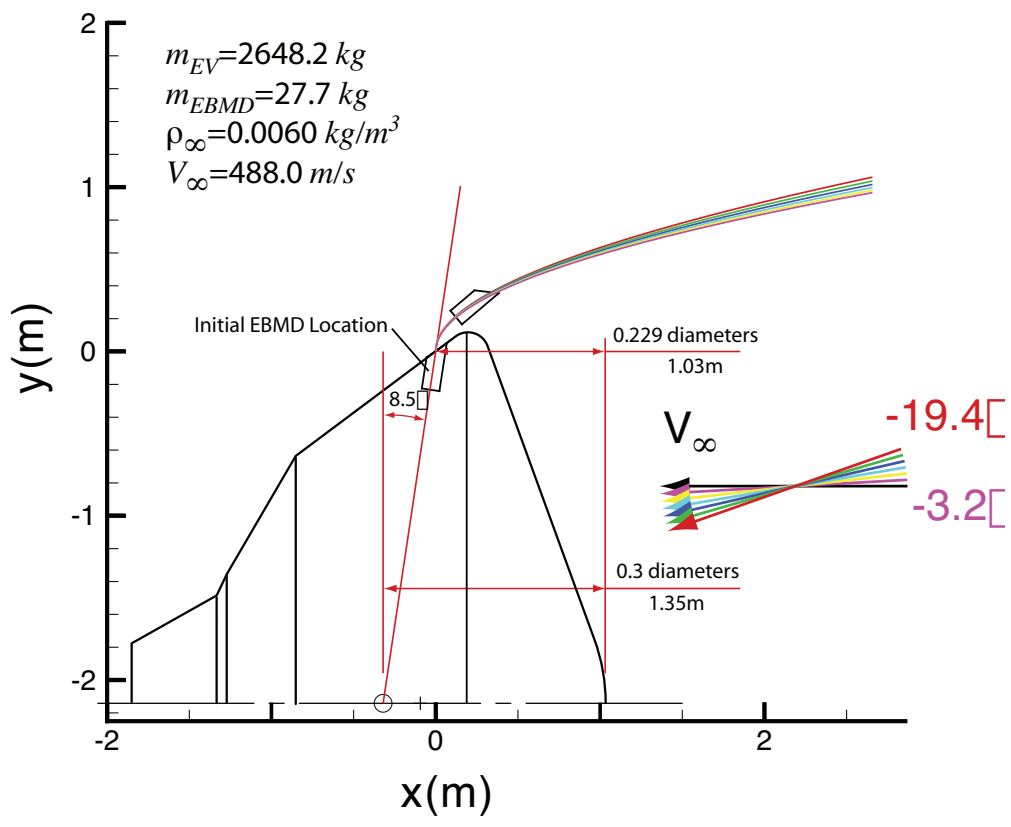

Figure 16. Planar EBMD separation simulations at conservative initial conditions

The second analysis took conservative density, angle-of-attack and mass property values for jettison conditions and simulated the EBMD departure with an unsteady CFD solution. The cases were run using the NASA OVERFLOW CFD code, version $2 .{ }^{6}$ This analysis was to add higher fidelity analysis to support the planar simulation and investigate whether any dynamics could be introduced by aerodynamic forces that might cause recontact concerns. 6-DoF dynamics of the EBMD were not captured in the planar simulation, where the aerodynamics model consisted only of a constant drag coefficient.

The OVERFLOW CFD code was described briefly earlier. Note that the Baldwin-Barth one-equation turbulence model was used in this simulation. Figure 17 shows Mach contour plots at different times during the full time-accurate simulation, showing the departure of an EBMD. The capsule was held at $\alpha=0^{\circ}$, but 
was free to decelerate due to the dynamic pressure acting on the body. The balance mass was released with a $1 \mathrm{~m} / \mathrm{s}$ separation velocity and is free to tumble. Rates are imparted on the EBMD as it slides out of its mount. Figure 18 shows a detail view of the EBMD as it passes around the shoulder at time step 2530.

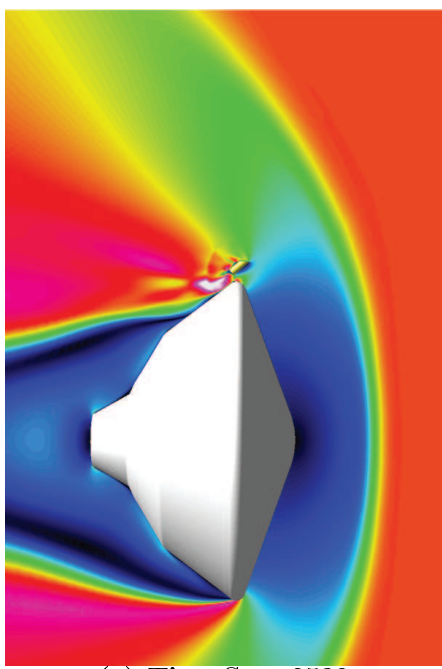

(a) Time Step 2530

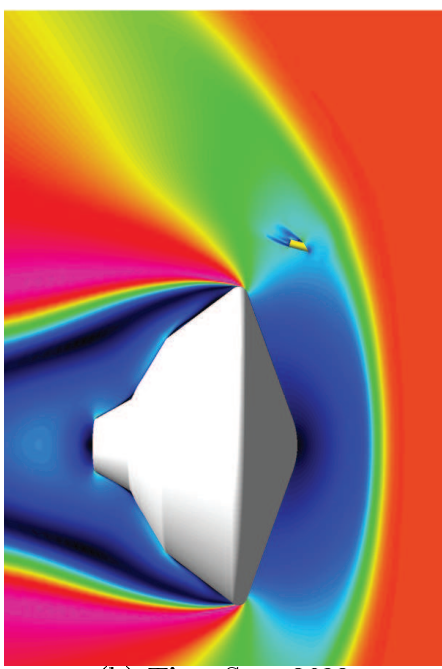

(b) Time Step 2680

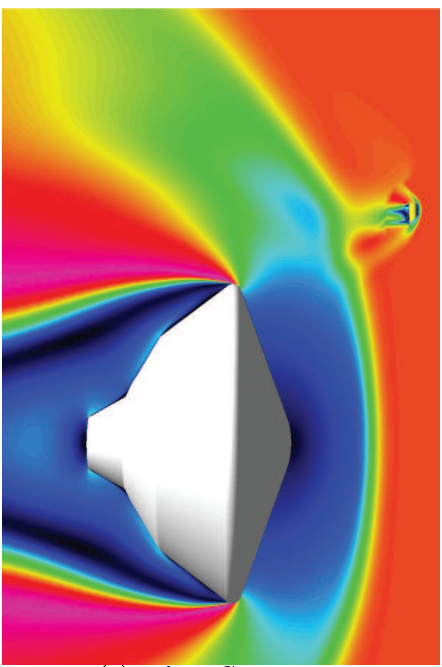

(c) Time Step 2850

Mach Countours

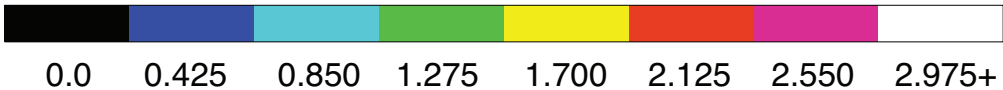

Figure 17. EBMD departure from OVERFLOW unsteady CFD solution

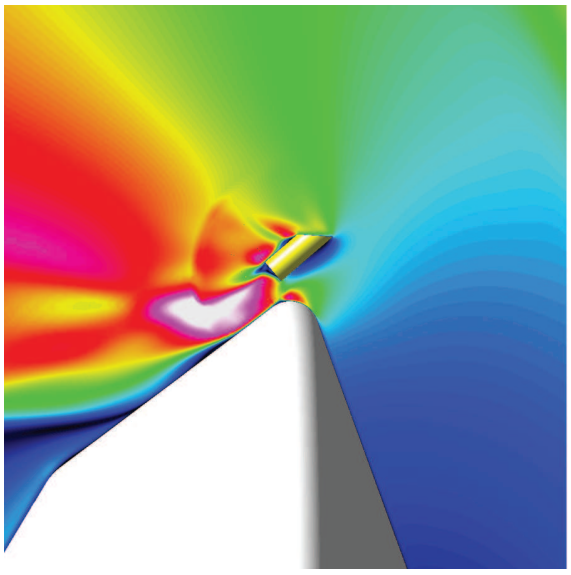

Figure 18. Detail of EBMD/Shoulder Region at time step 2530

Both the simple planar analysis and the multi-DoF OVERFLOW CFD simulation confirmed the initial analysis. The difference in ballistic coefficient ensures long term recontact and the aerodynamic effects near the EV do no significantly alter the balance mass trajectory or rotational motion while in the vicinity of the EV shoulder. This analysis shows the EBMD jettisons to be largely benign with little chance of recontact. However, this problem is a good example of the many new problems that MSL must investigate prior to flight. Any deviation from how earlier Mars missions have flown must be analyzed. The risk to mission success is too great to assume that a change is benign. The challenge for the project is to address each issue with the proper fidelity to retire the risk without draining resources from investigating the many other areas of concern. 


\section{6-DoF Uncertainty model}

In many ways, the uncertainty model used to bound the force and moment coefficients is the most critical part of the aerodynamic database. If the uncertainties properly bound the aerodynamic data and are implemented correctly, the data's use in simulation will properly identify trajectory or mechanical design changes necessary for a successful flight regardless of how accurately the data base is resolved. If the vehicle can fly with low fidelity data and very large uncertainties, there is no need for more accuracy or higher fidelity analysis or testing. Conversely, a high fidelity database might miss a critical aerodynamic property of a vehicle if the tolerances are too tight and don't account for unknown unknowns. For example, a database with extensive wind tunnel data might miss-predict key flight characteristics at Mars if the uncertainties bound only the experimental data and do not account for air $/ \mathrm{CO}_{2}$ differences. This section looks at some historical uncertainty models and then describes the MSL uncertainties, how they are based on those used for recent Mars missions, and how they have changed to account for the additional uncertainties introduced by lifting flight.

\section{Viking Uncertainties}

There is little documentation describing how the Viking uncertainty model was determined. The dispersions are generally smaller than those used in recent Mars missions. ${ }^{20}$ However, the manner in which these uncertainties were applied in simulation and how risk was assessed during the Viking project was not well documented either. The modern computational assets have fundamentally changed how risk assessment trajectory design is conducted. The use of Monte Carlo simulation to define landing ellipses and quantify risks was in its infancy during Viking, but has been used extensively during recent Mars missions. Therefore, MSL is basing uncertainty magnitudes on recent Mars missions which have a heritage chain dating back to Mars Pathfinder. The implementation of uncertainties require several modifications for the MSL entry vehicle as it is flying a lifting trajectory. The MER pitching moment uncertainty model provides a good case study, where using it directly with MSL would produce nonphysical pitching moment curves.

\section{MER Uncertainties}

The MER capsule was axisymmetric with no nominal radial cg offset and the application of the uncertainties was fairly straight forward. Pitching moment dispersions always act in the $\alpha_{T}$ plane:

$$
\left.C_{m_{T-D i s p}}\right|_{c g}=\left[\left.C_{m}\left(\alpha_{T}\right)\right|_{M R P}+\frac{\Delta x}{d} C_{N}\left(\alpha_{T}\right)\right]+U_{C_{m}}^{A}
$$

In Equation 2, $U_{C_{m}}^{A}$ is an adder that for MER had a $3 \sigma$ range of \pm 0.005 . Figure 19 shows a graphical representation of the uncertainty bounds and shows that an adder uncertainty implemented in this fashion actually creates nonphysical trim characteristics of the capsule. For a positive adder, the capsule trims at any combination of $\alpha$ and $\beta$ equal to the value of $\alpha_{T}$ where the dispersed $C_{m_{T}}$ value equals zero. For a negative adder, the capsule becomes "super-stable," never reaching a $C_{m_{T}}$ value of zero as there is no negative total angle-of-attack. In practice, this methodology is acceptable for axisymmetric shapes with no radial offset, especially one that is spinning. It was accepted as the approach for MER as a simple way to disperse the trim angle-of-attack. For non-spinning vehicles, or those with radial cg offsets, this approach may result in unrealistic flight characteristics and incorrectly influence Monte Carlo results.

\section{Moment Reference Point for Uncertainties}

One fundamental assumption of the MSL dispersion model is that moment uncertainties should be applied at the center-of-gravity. If they are not, the force uncertainties (axial, normal and side forces) will be reflected in the moments when they are transferred from an arbitrary moment reference point to the centerof-gravity. The correlated variation of moments and forces could possibly be identified through extensive wind tunnel data and possibly CFD analysis to account for effects not properly captured in experimental facilities. This analysis of the coupled variation of forces and moments could introduce specific center-ofgravity variations that better reflect the true uncertainties in aerodynamics model. The amount of wind tunnel data to do so would be prohibitive and the determination of correlated uncertainties with CFD 


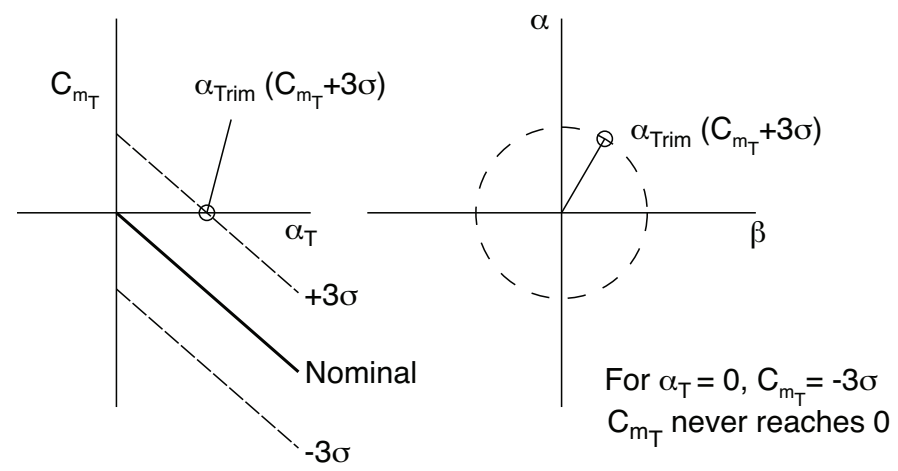

Figure 19. MER $C_{m}$ uncertainty implementation

data is beyond the computational state-of-the-art. Without such understanding, uncertainties are best applied at the center of gravity, thus decoupling the dispersions placed on each aerodynamic coefficient. The magnitude and functional form of the uncertainties applied to each coefficient can then be conservatively determined/bounded with available data and engineering analysis independent of the other coefficients.

\section{MSL Uncertainties}

The MSL uncertainties for the static aerodynamic coefficients are presented in Table 2 with values used in two prior missions. The drag uncertainties were updated slightly, with changes supported by MPF flight results. The flight regimes were also realigned slightly to be consistent with different computational and experimental methods used to generate data in each regime. MSL decomposes the uncertainties into six components and has added multipliers. The implementation of the MSL uncertainties is detailed below, followed by a more detailed description of these changes to the uncertainty model. The changes reflect an attempt to improve upon earlier models, and to account for additional uncertainties associated with lifting flight.

Table 2. Static Aerodynamics Uncertainties

\begin{tabular}{|c|c|c|c|c|c|}
\hline $\mathrm{MPF}$ & $C_{A}$ & $C_{N}$ & $C_{m}$ & $C_{n}$ & $C_{l}$ \\
\hline Mach $>12^{*}$ & $\pm 2 \%$ & \pm 0.01 & \pm 0.003 & $N A$ & $N A$ \\
\hline Mach $<8^{*}$ & $\pm 10 \%$ & \pm 0.01 & \pm 0.005 & $N A$ & $N A$ \\
\hline \multirow[t]{2}{*}{ MER } & & & & & \\
\hline & $C_{A}$ & $C_{N}$ & $C_{m}$ & $C_{n}$ & $C_{l}$ \\
\hline Knudsen $>0.1$ & $\pm 5 \%$ & \pm 0.01 & \pm 0.005 & $N A$ & $N A$ \\
\hline Mach > 10* & $\pm 3 \%$ & \pm 0.01 & \pm 0.003 & $N A$ & $N A$ \\
\hline Mach $<5^{*}$ & $\pm 10 \%$ & \pm 0.01 & \pm 0.005 & $N A$ & $N A$ \\
\hline \multirow[t]{2}{*}{ MSL } & & & & & \\
\hline & $C_{A}$ & $C_{N}, C_{Y}$ & $C_{m}$ & $C_{n}$ & $C_{l}$ \\
\hline Knudsen $>0.1$ & $\pm 5 \%$ & $\pm 0.01, \pm 10 \%$ & $\pm 0.005, \pm 20 \%$ & $\pm 0.005, \pm 20 \%$ & 0.0005 \\
\hline Mach $>10^{*}$ & $\pm 3 \%$ & $\pm 0.01, \pm 10 \%$ & $\pm 0.006, \pm 20 \%$ & $\pm 0.003, \pm 20 \%$ & 0.000219 \\
\hline Mach $<5^{*}$ & $\pm 10 \%$ & $\pm 0.01, \pm 10 \%$ & $\pm 0.005, \pm 20 \%$ & $\pm 0.005, \pm 20 \%$ & 0.00023 \\
\hline
\end{tabular}

* Uncertainties are linearly blended between regimes 
The static dispersion models are given by the following equations:

$$
\begin{aligned}
& C_{A_{D i s p}}=C_{A}(\alpha, \beta)\left(1+U_{C_{A}}^{M}\right) \\
& C_{N_{D i s p}}=\left[C_{N}(\alpha, \beta)+U_{C_{N}}^{A}\right]\left(1+U_{C_{N}}^{M}\right) \\
& C_{Y_{\text {Disp }}}=\left[C_{Y}(\alpha, \beta)+U_{C_{Y}}^{A}\right]\left(1+U_{C_{N}}^{M}\right) \\
& \left.C_{m_{D i s p}}\right|_{c g}=\left[\left.C_{m}(\alpha, \beta)\right|_{M R P}+\frac{\Delta x}{d} C_{N}(\alpha, \beta)-\frac{\Delta z}{d} C_{A}(\alpha, \beta)+U_{C_{m}}^{A}\right]\left(1+U_{C_{m}}^{M}\right) \\
& \left.C_{n_{D i s p}}\right|_{c g}=\left[\left.C_{n}(\alpha, \beta)\right|_{M R P}+\frac{\Delta x}{d} C_{Y}(\alpha, \beta)+\frac{\Delta y}{d} C_{A}(\alpha, \beta)+U_{C_{n}}^{A}\right]\left(1+U_{C_{n}}^{M}\right) \\
& \left.C_{l_{D i s p}}\right|_{c g}=\frac{\Delta y}{d} C_{N}(\alpha, \beta)-\frac{\Delta z}{d} C_{Y}(\alpha, \beta)+U_{C_{l}}^{A}
\end{aligned}
$$

Where $U_{C_{x}}^{M}$ and $U_{C_{x}}^{A}$ are the MSL multiplier and adder dispersions. The $3 \sigma$ limits of these adders and multipliers are listed in Table 2. A constant adder, $U_{C_{l}}^{A}$, is applied to the roll torque at the center of gravity; the nominal roll torque about the moment reference point is zero. When these dispersion models are applied in a 6-DoF Monte Carlo analysis, terms are applied randomly (one value for each run) between the positive and negative $3 \sigma$ limits listed in Table 2 using a Gaussian distribution. For each flight regime, there is one dispersion value selected randomly for each uncertainty variable and held constant for each run.

The magnitudes of the Mars Pathfinder dispersions were selected by project aerodynamicists and the NASA Langley hypersonic aerodynamics community, based on available data (limited CFD solutions and Viking wind tunnel data) and engineering judgment. Gaussian distributions were used to disperse the aerodynamics even though there were insufficient data to properly define the standard deviations of the available computational or experimental data. While some contributions to the uncertainties could be quantified (wind tunnel uncertainties, CFD grid sensitivities, etc.) the dominant uncertainty remained as unknown unknowns in applying computational solutions and ground based experimental data to a real vehicle flying at Mars with geometric imperfections, ablation, etc. The Gaussian distribution best reflected the reasonable confidence in the nominal aerodynamic data, while trying to account for physical phenomena or conditions at Mars that the aerodynamicists had missed. It should be noted that the use of Gaussian distributions based on engineering judgment produces statistical results in Monte Carlo simulations of fidelity only equal to that of the judgment. Engineering judgment must therefore be exercised in the interpretation of the simulation results. However, this approach has been successful in the design and prediction of recent Mars entries.

The magnitudes of MSL dispersions are based on those used in previous Mars missions. As the dispersions were decomposed into six components, the coefficients in the yaw plane adopted the corresponding value in the pitch plane. Three primary exceptions should be noted. First, an assessment of pitching moment dispersions at hypersonic speeds showed a sensitivity of $C_{m}$ to gas chemistry models and chemical reaction rates used in LAURA at non-zero trim angles-of-attack. The $C_{m}$ adder was doubled in the hypersonic regime (Figure 5). Second, multipliers designed to vary the local force and moment slopes were added to the uncertainty models. Applied at the $\mathrm{cg}$ after adders were applied, the change of slope of transverse forces and pitch and yaw moments at the trim point will help assess the controller design. The multiplier values of 10 and $20 \%$ are loosely based on pitch and yaw frequency data extracted from previous missions. These values will be increased above current levels for stress testing the controller. The last significant change is the addition of a persistent roll torque dispersion. Simulations have shown that the controller is sensitive to an unpredicted, persistent rolling moment. The next section is devoted to describing the problems that have been identified and how the roll dispersion was quantified.

Finally, a note on dynamic stability. The pitch and yaw damping uncertainties have not been addressed in this section. Simulations have shown that the controller keeps rates low, minimizing destabilizing moments. Stress testing using constant positive pitch and yaw damping coefficients have shown the controller to be very robust to dynamic instabilities. Refer to Schoenenberger et $\mathrm{al}^{21}$ for an overview of the MSL dynamic stability testing and the conservative dispersion model used in simulation. 


\section{E. Roll torque dispersions}

In addition to the section describing the static aerodynamics uncertainty model, this section is devoted to one static aerodynamic variable. Simulations to date show that the MSL controller can be sensitive to roll torque dispersions. This section describes the problems encountered when the EV experiences large roll torque dispersion and the analysis done to quantify the uncertainties more carefully.

\section{Roll torque problems identified}

In early simulations, uncertainties were applied at the axial cg location, but the radial cg moment transfer was done after dispersions were applied to the aerodynamics. Monte Carlo simulations of the MSL entry showed that aerodynamic dispersions could produce large persistent roll torques which might confuse the control algorithms and cause the vehicle to deviate from the guidance flight path, missing the intended landing ellipse. The MSL controller has no way of identifying and correcting for a persistent roll torque explicitly. In practice, the controller tended to fail when a bank reversal was commanded by the guidance algorithm near peak dynamic pressure. The persistent roll torque in these simulations arose from large dispersions in yawing moment and side force. With a cg offset in the pitch plane, any side force produces a roll torque. This effect is analogous to a hang glider pilot shifting her weight to the left or right in order to roll the glider.

It was determined that an error in the dispersion model was producing the large roll torques. Dispersions were applied at the axial cg location, then transferred the radial offset distance. The dispersed side-force was exacerbating the roll torque in some cases. Correctly applying dispersions at the cg eliminated the dispersed side force contribution, reducing the roll torque dispersions and no controller failures occurred. However, the "incorrect" dispersion model had shown that the controller was susceptible to a persistent roll in some cases. In fact, this has been the only failure to be identified that is caused solely by the dispersed aerodynamics. Knowing the controller's sensitivity to persistent roll, it is critical to properly bound the roll torque dispersions. Roll torque dispersions had never been quantified for Mars entry vehicles before. First order engineering analysis was conducted to identify mechanisms by which the MSL heatshield could produce a persistent roll torque and to bound the physically possible limits of those mechanisms, thus conservatively bounding the roll torque dispersions.

\section{Roll torque from heatshield surface features}

An axisymmetric spacecraft with no protrusions will not have any roll torques at a trimmed orientation. This applies to vehicles with the center of gravity located on the spin-axis as well as those with a radial $\mathrm{cg}$ offset. The spin axis and vector from the axial cg location to the offset center of gravity offset defines a plane of symmetry. For a statically stable spacecraft, one can define the $\alpha, \beta$ coordinate system such that angle-of-attack oscillations occur in the defined pitch plane while sideslip oscillations are in the orthogonal plane. If the capsule is at a sideslip angle, the resulting side force, acting through the center of pressure will produce a roll torque. As the capsule is stable, it will oscillate in sideslip and the roll torques will oscillate about a mean zero roll torque.

Deviations from a perfectly symmetric body of revolution can result in a net persistent roll torque. A damaged heatshield can produce a non-zero trimmed sideslip angle, out of plane from the cg-defined pitch plane. This is the "hang glider" mechanism described earlier. However, for a $70^{\circ}$ sphere-cone the pressure distribution on a damaged heatshield that produces a non-zero trim angle-of-sideslip tends to produce less side force, mitigating much of the roll torque that would have been produced by an undamaged vehicle at the same orientation (though not trimmed). This suggests that the side force and yawing moment dispersions should not stack up to produce excessive roll torques together.

Another geometry variation that can effectively produce persistent roll torques is an asymmetric distribution of forward facing steps or defects on the capsule forebody. MSL's is the first non-monolithic heatshield to be flown to Mars. It is made up of many Phenolic Impregnated Carbon Ablator (PICA) tiles, attached to an aluminum honeycomb substructure. The gaps between tiles are filled with RTV. This tile assembly allows new mechanisms for roll torque production. Any combination of gap fillers rising above the OML, steps left by spalling heatshield material, ablated or eroded heatshield tiles or seams can slow oncoming flow, recovering pressure on the surfaces which add up to a net torque about the center of gravity. The greatest potential 
for a roll torque occurs at peak dynamic pressure. Based on aerodynamic analysis of different roll producing changes to the OML, this "pinwheel" mechanism appears to have the greatest potential for producing persistent roll torques. A first order analysis was performed to look at worst case surface features and approximate the possible pressure recovery on those features (using several different engineering assumptions) that would produce persistent roll torques. The analysis was intended to provide a roll torque dispersion value based on the limits of what is physically possible for the MSL heatshield, using very conservative assumptions. Had the physically possible limits produced roll torques even greater than those seen in earlier failed simulations, higher fidelity analysis would have been applied to this problem.

\section{Pressure recovery on a forward facing step}

There has been extensive work looking at drag augmentation to aircraft and entry vehicles due to surface imperfections. These surface features can be due to fabrication inaccuracies, ablation of non-homogeneous materials like carbon fiber, spallation or other kinds of damage. For the MSL heatshield, made up of many PICA tiles with seams filled by RTV, arc jet testing has shown that the RTV can ablate at a lower rate than the PICA field material, resulting in up to 0.2 inch steps above the remaining PICA heatshields. ${ }^{22}$ The aerodynamic effects on such a step can be approximated by a drag model developed from wind tunnel tests of a forward facing step. ${ }^{23-26}$ Those tests showed a good correlation between the mean dynamic pressure across the height of the step and the drag force acting on the step for a wide range of Mach numbers and step heights, normalized by the displacement thickness, $\delta^{*}$.

$$
D_{\text {step }}=C_{D} \bar{q} h l
$$

where

$$
\bar{q}=\frac{1}{2} \int_{o}^{h} \rho V^{2} d y
$$

Figure 20 shows how the drag coefficient collapses to a constant value for all but the smallest step heights. This data forms a starting point for the analysis of the MSL forebody pressure distribution.

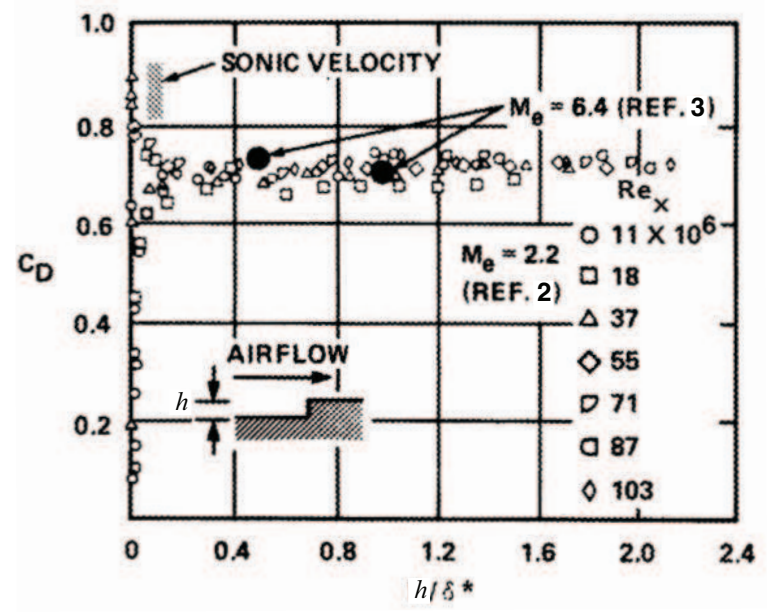

Figure 20. Drag coefficient for single forward-facing step ${ }^{26}$

There are several caveats to be made when applying this data to the MSL configuration, most having to do with the potential flow differences between a 2-D step in a wind tunnel and the complex 3-D flowfield over the MSL heatshield. However, the Mach independence shown in Figure 20 suggests that the details of the local flowfield are a second order effect. The drag produced when the flow is not aligned perpendicular to the step is not addressed in the wind tunnel data, but must be accounted for when estimating the roll torques produced on the MSL heatshield. The approach taken in this analysis is to use only the velocity 
component normal to the step when calculating the drag acting on the step. This approach is analogous to pressure calculations using Newtonian methods and velocity changes across oblique shocks.

Arc jet tests of the PICA heatshield material with RTV seams has shown that a step can develop as a heatshield ablates/erodes, leaving RTV standing proud above the remaining PICA material. A conservative upper limit of 0.2 inches was identified in the arc jet testing and is used as the conservative step height in this analysis.

This step height is small compared to the boundary layer thickness over much of the heatshield. Therefore, the average Mach number across the step height is subsonic over much of the surface. The experimental data does not measure drag for subsonic cases. In fact, the correlations presented by Czarnecki suggest that the constant $C_{D}$ correlation breaks down when the velocity in the boundary layer at the edge of the step height approaches Mach 1 and the step is a small fraction of the displacement thickness. Therefore, an additional method was used to predict the drag acting on the forward facing steps; the total pressure recovery was calculated. In addition to the experimental drag coefficient $\left(C_{D}=0.7\right.$.) with the mean approaching dynamic pressure (calculated using the normal component of velocity), the isentropic compression or Rayleigh-Pitot equation (depending on mean Mach number across the step height) is used to calculate the total pressure recovery on the step face. These two methods of calculating drag acting on the steps give two reasonable order-of-magnitude approximations to the actual pressure recovered. The pressure recovery method should bound the possible pressure recovery possible from the mean flow conditions across the specified step height. Table 3 shows the two drag equations and two integrals for calculating the mean approaching flow field normal to the step:

Table 3. Pressure Recovery Models

\begin{tabular}{cccc}
\hline Case & Mean Flow Conditions & Drag Force & 7-Ray $C_{l}$ \\
\hline \hline 1 & $\bar{q}_{n}=\frac{1}{2 h} \int_{0}^{h} \rho V_{n}^{2} d y=\frac{1}{2} \int_{0}^{h} \rho(V \sin \theta)^{2} d y$ & $D=C_{D} \bar{q}_{n} h l$ & 0.00007 \\
2 & $\bar{M}_{n}=\frac{1}{h} \int_{0}^{h} M_{n} d y=\frac{1}{h} \int_{0}^{h} M \sin \theta d y$ & $D=\left(P_{o}\left(\bar{M}_{n}\right)-P_{\text {wall }}\right) h l$ & 0.00010 \\
& & & \\
\hline
\end{tabular}

\section{Local flowfield and pressure recovery}

The surface distribution of the contribution to roll torque calculated using the relations in Table 3 , based on the near-wall flow conditions of a LAURA CFD solution at a peak dynamic pressure trajectory-point (Mach 18.1) are plotted in Figure 21. The mean conditions calculated for the cases are across a theoretical step height of $0.00508 \mathrm{~m}$ (0.20 in.). Basing torque on the theoretical pressure recovery produced by slowing down the mean normal Mach across a local step face produces a higher roll torque than calculating a force on the face using an empirical $C_{D}$ value of 0.7 and the mean dynamic pressure across the face. However, both methods of calculating forces on a step show very similar distributions over the MSL forebody, and the largest contribution to roll torque would be produced on any steps occurring nearly parallel to the positive or negative y axes. The lower forces calculated using the empirical drag coefficient may reflect the relaxation permitted the flow as much of the approaching flow would divert up and over a forward facing step.

\section{Worst-case step distribution}

For a conservative limit on the distribution of steps on the MSL PICA heatshield it is assumed that one half of the heatshield has the largest step height due to ablation seen in arc jet testing $(h=0.20 \mathrm{in}$.) along all radial seams. The pressure recovery models are only applied to the steps that are directed radially out from the EV centerline. Asymmetric pressures on the circumferential seams will not produce direct roll torques. Pressures on those seams could cause a shift in the trimmed angle-of-sideslip. The resulting sideforce would produce a persistent roll dispersion. However, this effect is much smaller than a similar pressure recovery on the radial seams.

The radial seam pattern of the MSL entry vehicle is approximated by 7 radial seams that start at the heatshield centerline and extend to the outer shoulder tiles. These seams were placed at $\phi=10^{\circ}, 26^{\circ}, 55^{\circ}, 83^{\circ}, 118^{\circ}, 148^{\circ}$ 


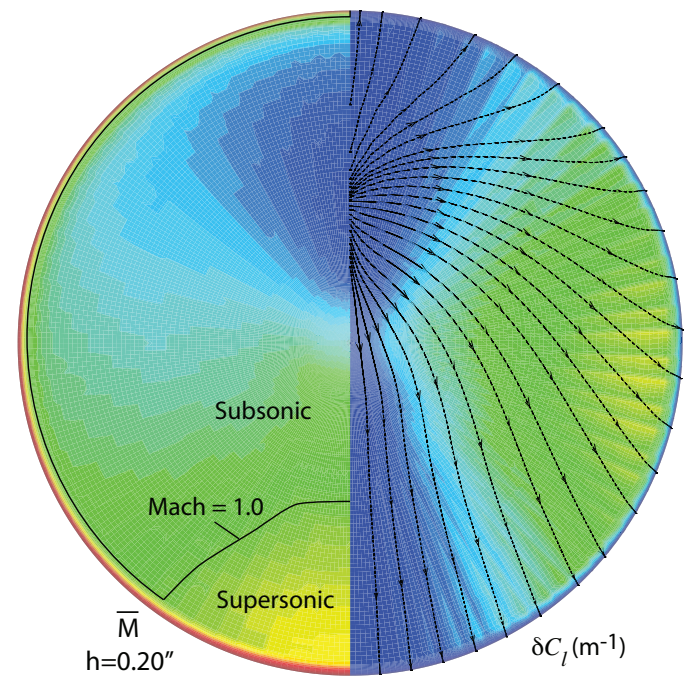

(a) Case 1

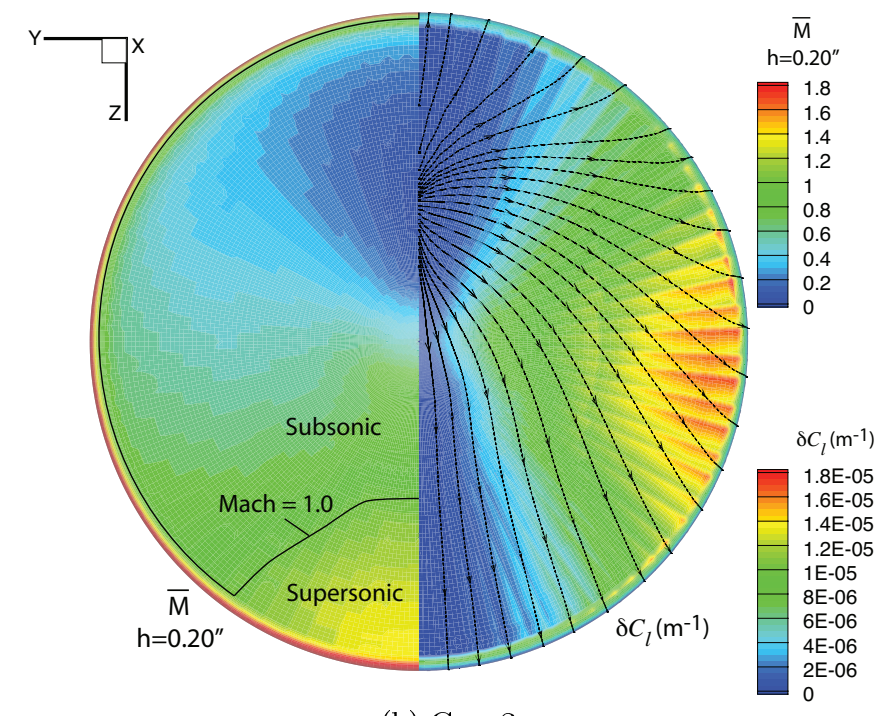

(b) Case 2

Figure 21. Plot of local $C_{l}$ contribution (per unit length) using empirical drag and pressure recovery models

and $168^{\circ}$, where $\phi=0^{\circ}$ corresponds to the pitch plane on the lee-side (opposite the stagnation region). The shoulder tile radial seams are approximated by 16 rays starting at $\phi=6^{\circ}$ and then occurring every $11^{\circ}$ circumferentially around to the stagnation side of the heatshield. Figure 22 shows these rays overlayed on the PICA tile pattern of the MSL heatshield. Note that the rays pass roughly along radial seam paths on the cone of the heatshield, but occasionally pass over an annular tile ring with no corresponding radial seam or conversely miss some of the radial seams on the outer annulus near the shoulder. These rays approximating the actual tile pattern are reasonable. The reduction in fidelity is small compared to the overriding conservative assumption steps developing on one side of the pitch plane only.

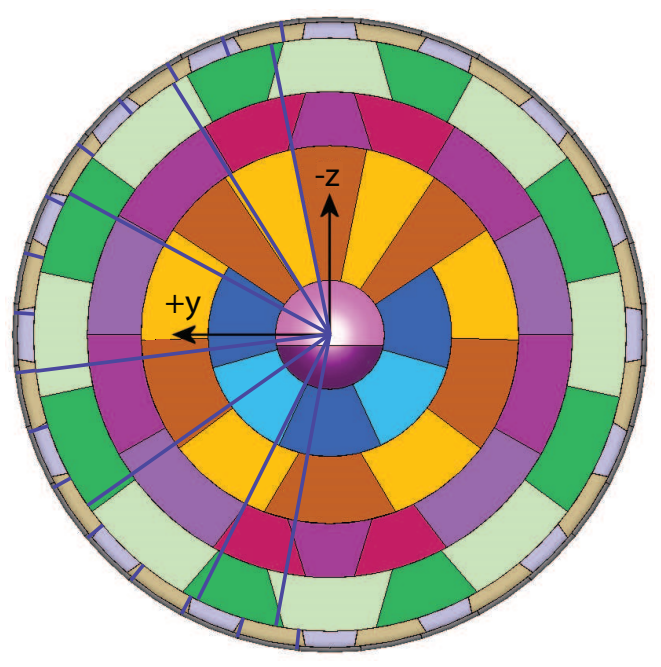

Figure 22. Rays used to approximate radial steps on PICA heatshield

Using the two pressure recovery models, the torques calculated along the rays in Figure 22 sum to produce the values shown in Table 3. The contributions from individual rays are shown in Figure 23. These calculations show that the largest contribution occurs on the steps out to the side of the heatshield (out of the pitch plane). The high dynamic pressure on the lee-side is a more energetic flow that when slowed could recover more pressure. However, the flow near the pitch plane of symmetry is essentially tangent 
to any radial seam in these areas. Thus, there is little torque generated by steps occurring in the highest dynamic pressure regions. The individual contributions to roll torque from the steps out at the shoulder are considerably less than steps in the $\phi=60^{\circ}-120^{\circ}$ range of the cone region. The seam lengths are much longer on the cone, which accounts for the larger contribution. However, there are many more seams along the shoulder and any pressure on a radial seam will have the largest moment arm out at the shoulder. The small shoulder radius creates a high shear environment and the development of larger steps or defects may be more likely. The shoulder geometry may permit spalling not scene in arc jet testing or might be susceptible to other damage that propagates or creates steps greater than the assumed 0.20 inches.

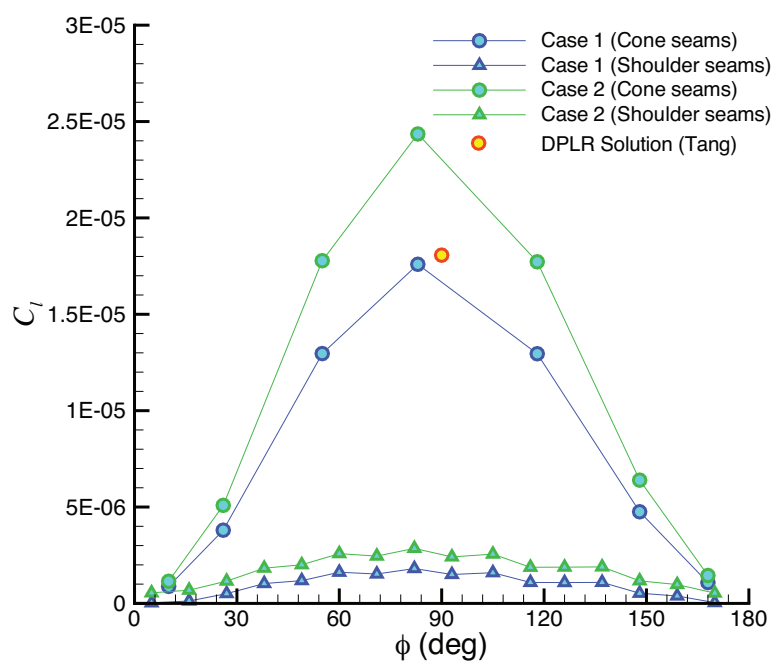

(a) Pressure recovery using velocity normal to step

Figure 23. Torque contributions from individual rays calculated using emprical drag and pressure recovery models

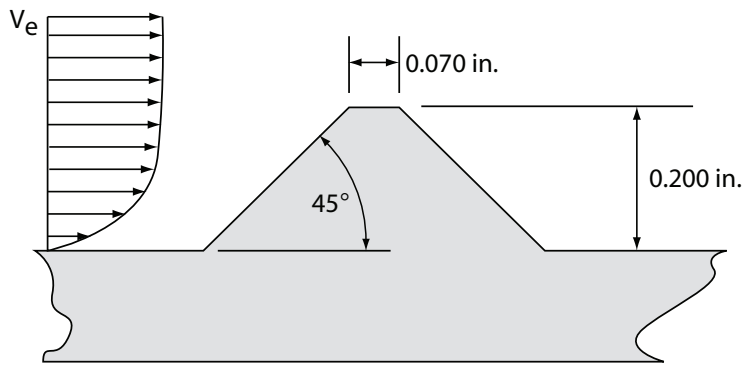

Figure 24. Cross-section of RTV step modeled in CFD solution

One DPLR solution was run by Chun Tang at NASA Ames to provide a higher fidelity data point for comparison with the first order engineering methods. The solution included a protrusion with the step height matching that used in this analysis. The protrusion has a trapezoidal cross-section and is canted $45^{\circ}$ away from the approaching flow. The resulting roll torque from that solution has been added to Figure 23 and shows very close agreement with Case 1 . This is not a direct validation of the methodology of Case 1 as the data used to produce the Case 1 result was for a $90^{\circ}$ forward facing step. The $45^{\circ}$ forward facing step of in Tang's solution should produce more relaxation and less pressure recovery than a $90^{\circ}$ step. This suggests that Case 1 under-predicts the actual recovery a $90^{\circ}$ step might see. Overall, it appears that a $90^{\circ}$ step would actually fall somewhere between the two models used. This CFD spot check, while not validating the empirical analysis, does indicate that the empirical analysis is reasonable and that the current dispersions appear to be sufficiently conservative. The pressure recovery method of Case 3 is just slightly more conservative, compared to this spot check. The roll torque $3 \sigma$ dispersion magnitude in Table 2 is likely 
overly conservative, but not absurdly large compared to this first order analysis. The roll torque dispersions will be kept at their current values, but simulation results will be interpreted with an understanding of this conservatism.

\section{Conclusions}

Several of the key challenges of Mars entry new to the MSL entry vehicle have been presented. The vehicles that have landed on Mars have all provided information upon which MSL can claim some benefit. As MSL will be flying such a large and massive vehicle at trim angles greater than any before it, analysis and testing are required to ensure that MSL can safely fly in the expanded flight envelope. Some mechanical design decisions have presented new aerodynamics challenges. The RCS configuration has gone through many iterations to arrive at what analysis and testing are showing to be a good design. The EBMD jettison events are new to planetary entry missions. Their novelty alone requires that analysis be done to ensure no aerodynamic issues have been introduced.

Many CFD solutions, wind tunnel test points, and engineering calculations have been collected or calculated just to show that MSL will fly succesfully. This does not diminish the importance of the analyses. It is critical that the aerodynamics of the MSL capsule be understood at the flight conditions in which it will be flying. Where possible, simple but conservative bounding analysis, perhaps backed by higher fidelity spot checks, can assess a particular aerodynamic question and retire the risk. The EBMD jettison events and roll torque dispersions are two good examples. Even for areas where risk is low, the analysis leads to a more complete understanding of the aerodynamics of the MSL system. The benefit of this analysis may show up during flight operations where decisions caused by rapidly changing weather conditions leading up to entry may require fast analyses and decisions about changes to the timing of entry events. Having this analysis in hand will make sure the necessary quick decisions are based on good information.

\section{Acknowledgments}

The authors would like to acknowledge the computation contributions of Chun Tang. His CFD analysis provided invaluable support for the assessment of the roll torque dispersions.

\section{References}

${ }^{1}$ Lang, J. R. and Sarver, D. A., "Experimental Aerodynamic Characteristics of the Viking Lander Capsule During Aeroshell Staging at M = 0.3 to 1.13," TR- 3720301, Martin Marietta Corporation, 1972.

${ }^{2}$ Cheatwood, F. M. and Gnoffo, P. A., "User's Manual for the Langley Aerothermodynamic Upwind Relaxation Algorithm (LAURA)," NASA TM 4674, 1996.

${ }^{3}$ Riley, C. and Cheatwood, F., "Distributed-Memory Computing with the Langley Aerothermodynamic Upwind Relaxation Algorithm (LAURA)," Advances in Engineering Software, Vol. 29, No. 3-6, July 1998, pp. 317-324.

${ }^{4}$ Chan, W. M., Gomez III, R. J., Rogers, S. E., and Buning, P. G., "Best Practices in Overset Grid Generation," AIAA 2002-3191, 2002.

${ }^{5}$ Chaderjian, N. M. and Olsen, M. E., "CEV CFD Simulation Guidelines for the OVERFLOW 2 Navier-Stokes Solver," NASA/EG-CAP-06-33, Version 1.2, August 31, 2004.

${ }^{6}$ Buning, P. G., Gomez, R. J., and Scallion, W. I., "CFD Approaches for Simulation of Wing-Body Stage Separation," AIAA 2004-4838, 2004.

${ }^{7}$ Murphy, K. J., Borg, S. E., Watkins, A. N., Cole, D. R., and Schwartz, R. J., "Testing of the Crew Exploration Vehicle in NASA Langley's Unitary Plan Wind Tunnel," AIAA 2007-1005, 2007. 1998.

${ }^{8}$ Micol, J. R., "Langley Aerothermodynamic Facilities Complex: Enhancements and Testing Capabilities," AIAA 98-0147,

${ }^{9}$ Polutchko, R. J., "Viking entry aerodynamics and heating," Proceedings of Outer Planet Probe Technology Workshop, Sect. 1 through 11, 75N20383, April 1974.

${ }^{10}$ Gnoffo, P. A., Braun, R. D., Weilmuenster, J. K., Mitcheltree, R. A., Engelund, W. C., and Powell, R. W., "Prediction and Validation of Mars Pathfinder Hypersonic Aerodynamic Database," Journal of Spacecraft and Rockets, Vol. 36, No. 3, May-June 1999, pp. 367-373.

${ }^{11}$ Schoenenberger, M., Cheatwood, F., and Desai, P., "Static Aerodynamics of the Mars Exploration Rover Entry Capsule," AIAA 2005-0056, January 2005.

${ }^{12}$ Desai, P. N., Prince, J. L., Queen, E. M., and Cruz, J. R., "Entry, Descent and Landing Performance of the Mars Phoenix Lander," AIAA 2008-7346, 2008. 
${ }^{13}$ Camac, M. and ed. Hall J.G., "CO2 Relaxation Process in Shock Waves," Fundamental Phenomena in Hypersonic Flow, Cornell Univ. Press, Ithaca, New York, 1966.

${ }^{14}$ Millcan, R. C. and White, D. R., "Systematics of Vibrational Relaxation," Journal of Chemical Physics, Vol. 39, No. 12, pp. 3209-3213.

${ }^{15}$ Anderson, W. K. and Bonhaus, W. K., "An Implicit Upwind Algorithm for Computing Turbulent Flows on Unstructured Grids," Computers Fluids, Vol. 23, No. 1, 1994, pp. 1-21.

${ }^{16}$ A., D. A., Glass, C. E., Edquist, K. T., Schoenenberger, M., Chwalowski, P., Van Norman, J., Scallion, W. I., Tang, C., Wright, M. J., Cheatwood, F. M., Hollis, B. R., Lessard, V. R., and Takashima, N., "Design Considerations for Reaction Control Systems," 5th International Planetary Probe Workshop, Bordeaux, France, June 25-29 2007.

${ }^{17}$ Dyakonov, A. A., Schoenenberger, M., Scallion, W. I., Van Norman, J., Novak, L., and Tang, C., "Aerodynamic Interference Due to MSL Reaction Control System," AIAA 2009-3915, 2009.

${ }^{18}$ Scallion, W. I., Compton, H. R., Suit, W. T., Powell, R. W., Blackstock, T. A., and Bates, B. L., "Space Shuttle Third Flight (STS-3) Entry RCS Analysis," AIAA 83-0116, January 1983.

${ }^{19}$ Salas, M. D., "The Numerical Calculation of Inviscid Plume Flow Fields," AIAA 74-523, June 1974.

${ }^{20}$ Flaherty, T. M., "Aerodynamics Data Book," TR- 3709014, Martin Marietta Corporation, 1972.

${ }^{21}$ Schoenenberger, M., Yates, L., and Hathaway, W., "Dynamic Stability Testing of the Mars Science Laboratory Entry Capsule," AIAA 2009-3917, 2009.

${ }^{22}$ Beck, R., Driver, D., Wright, M., Laub, B., Slimko, E., and Edquist, K., "Development of the Mars Science Laboratory Heatshield Thermal Protection System," AIAA 2009-4229, 2009.

${ }^{23}$ Czarnecki, K. R., Jackson, M. W., and Monta, W. J., "Studies of Skin Friction at Supersonic Speeds.Converence on Supersonic-Transport Feasibility Studies and Supporting Research," NASA TMX-905, NASA Langley Research Center, 1963, pp. 177-189.

${ }^{24}$ Czarnecki, K. R., "The Problem of Roughness Drag at Supersonic Speeds," NASA TND-3589, NASA Langley Research Center, 1966. 1981.

${ }^{25}$ Nestler, D. E., "An Experimental Study on Cavity Flow on Sharp and Blunt Cones at Mach 8," AIAA 81-0335, January

${ }^{26}$ Szostowski, D. J., Nestler, D. E., and Lowe, D. L., "Viscous Code Prediction of Re-entry Vehicle Roll Torque Based on Ablated Surface Topology," AIAA 82-0901, June 1982. 